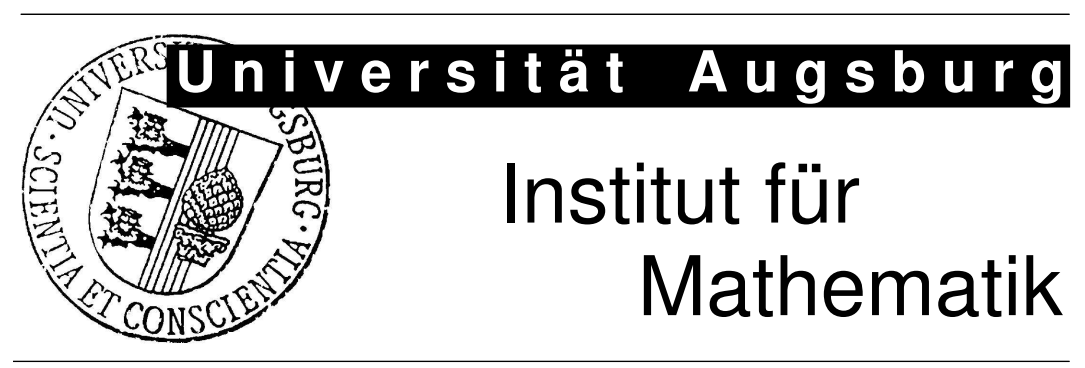

Carsten Carstensen, Ronald H.W. Hoppe, Natasha Sharma, Tim Warburton Adaptive Hybridized Interior Penalty Discontinuous Galerkin Methods for H(curl)-Elliptic Problems 


\section{Impressum:}

\section{Herausgeber:}

Institut für Mathematik

Universität Augsburg

86135 Augsburg

http://www.math.uni-augsburg.de/pages/de/forschung/preprints.shtml

\section{ViSdP:}

Ronald H.W. Hoppe

Institut für Mathematik

Universität Augsburg

86135 Augsburg

Preprint: Sämtliche Rechte verbleiben den Autoren (C) 2010 


\title{
ADAPTIVE HYBRIDIZED INTERIOR PENALTY DISCONTINUOUS GALERKIN METHODS FOR H(CURL)-ELLIPTIC PROBLEMS
}

\author{
C. CARSTENSEN*†, R. H. W. HOPPE ${ }^{\ddagger} \S$, N. SHARMA ${ }^{\ddagger}$ AND T. WARBURTON $₫ \|$
}

\begin{abstract}
We develop and analyze an adaptive hybridized Interior Penalty Discontinuous Galerkin (IPDG-H) method for $\mathrm{H}$ (curl)- elliptic boundary value problems in 2D or 3D arising from a semi-discretization of the eddy currents equations. The method can be derived from a mixed formulation of the given boundary value problem and involves a Lagrange multiplier that is an approximation of the tangential traces of the primal variable on the interfaces of the underlying triangulation of the computational domain. It is shown that the IPDG-H technique can be equivalently formulated and thus implemented as a mortar method. The mesh adaptation is based on a residual-type a posteriori error estimator consisting of element and face residuals. Within a unified framework for adaptive finite element methods, we prove the reliability of the estimator up to a consistency error. The performance of the adaptive symmetric IPDG-H method is documented by numerical results for representative test examples in $2 \mathrm{D}$.
\end{abstract}

Key words. adaptive hybridized Interior Penalty Discontinuous Galerkin method, a posteriori error analysis, $\mathrm{H}$ (curl)-elliptic boundary value problems, semi-discrete eddy currents equations

AMS subject classifications. $65 \mathrm{~F} 10,65 \mathrm{~N} 30$

1. Introduction. Discontinuous Galerkin (DG) methods are widely used algorithmic schemes for the numerical solution of partial differential equations (PDE). For a comprehensive description, we refer to the survey article [24] and the references therein. As far as elliptic boundary value problems are concerned, DG methods can be derived from a primal-dual mixed formulation using local approximations of the primal and dual variables by polynomial scalar and vector-valued functions and appropriately designed numerical fluxes. Among the most popular schemes are Interior Penalty DG (IPDG) and Local DG (LDG) methods which have been analyzed by means of a priori estimates of the global discretization, e.g., in [3, 5, 23, 39]. For H(curl)-elliptic boundary value problems arising from a semi-discretization of the eddy currents equations, symmetric IPDG methods have been studied in [36]. The time-harmonic Maxwell equations have been addressed in [46].

On the other hand, the a posteriori error analysis and application of adaptive finite element methods (FEM) for the efficient numerical solution of boundary and initialboundary value problems for PDE has reached some state of maturity as documented by a series of monographs. There exist several concepts including residual and hierarchical type estimators, error estimators that are based on local averaging, the so-called goal oriented dual weighted approach, and functional type error majorants (cf. $[2,6,7,30,44,49]$ and the references therein). A posteriori error estimators for DG methods applied to second order elliptic boundary value problems have been developed and analyzed in $[1,11,18,38,40,47]$. In particular, a convergence analysis of adaptive symmetric IPDG methods has been provided in [12,34] and [41]. Residualand hierarchical-type a posteriori error estimator for $\mathrm{H}$ (curl)-elliptic problems have

\footnotetext{
${ }^{*}$ Dep. of Math., Humboldt Universität zu Berlin, D-10099 Berlin, Germany

${ }^{\dagger}$ Dep. of Comput. Sci. Eng., Yonsei University, Seoul 120-749, Korea

$\ddagger$ Dep. of Math., Univ. of Houston, Houston, TX 77204-3008, U.S.A.

$\S$ Inst. of Math., Univ. of Augsburg, D-86159 Augsburg, Germany

๑ CAAM, Rice Univ., Houston, TX 77005-1892, U.S.A.

$\|$ The first author has been supported by the German National Science Foundation DFG within the Research Center MATHEON and by the WCU program through KOSEF (R31-2008-000-100490). The other authors acknowledge support by the NSF grant DMS-0810176
} 
been studied in $[8,9,10,20,37]$. A convergence analysis for residual estimators has been developed in [19] for 2D and in [35] for 3D problems.

From a computational point of view, DG methods suffer from a relatively huge amount of globally coupled degrees of freedom (DOF) compared to standard FEM. Hybridization is a technique that gives rise to a significant reduction of the globally coupled DOF. It has been introduced for mixed FEM in [31] and further studied in $[4,13,15,25,26]$. Adaptive mixed hybrid methods on the basis of reliable a posteriori error estimators have been considered in [14, 45] and [50]. For DG methods, a survey of hybridized DG (DG-H) methods has been provided in [26], whereas a unified analysis has been developed in [28]. However, adaptive DG-H methods have not yet been investigated.

In this paper, we will derive and analyze a residual-type a posteriori error estimator for hybridized symmetric IPDG (IPDG-H) methods applied to H(curl)-elliptic boundary value problems in $3 \mathrm{D}$. The analysis will be carried out within a unified framework provided for adaptive finite element approximations in [17, 18, 20, 21, 22]. The paper is organized as follows: In Section 2, we introduce some basic notation and present the class of $\mathrm{H}(\mathrm{curl})$-elliptic boundary value problems to be approximated by symmetric IPDG-H methods. Section 3 deals with the development of symmetric IPDG-H methods based on a mixed formulation of the elliptic boundary value problems. We establish its relationship with mortar techniques which allows the implementation as a mortar method. In section 4, we present the residual-type a posteriori error estimator and prove its reliability. Finally, in section 5, we provide a detailed documentation of numerical results to illustrate the performance of the symmetric IPDG-H methods.

2. Basic Notations. Let $\Omega \subset \mathbb{R}^{3}$ be a simply connected polyhedral domain with boundary $\Gamma=\partial \Omega$ such that $\Gamma=\bar{\Gamma}_{D} \cup \bar{\Gamma}_{N}, \Gamma_{D} \cap \Gamma_{N}=\emptyset$. We denote by $\mathcal{D}(\Omega)$ the space of all infinitely often differentiable functions with compact support in $\Omega$ and by $\mathcal{D}^{\prime}(\Omega)$ its dual space referring to $\langle\cdot, \cdot\rangle$ as the dual pairing between $\mathcal{D}^{\prime}(\Omega)$ and $\mathcal{D}(\Omega)$. We further adopt standard notation from Lebesgue and Sobolev space theory. In particular, for a subset $D \subset \Omega$, we refer to $L^{2}(D)$ and $\mathbf{L}^{2}(D)$ as the Hilbert spaces of scalar and vector-valued square integrable functions with inner products $(\cdot, \cdot)_{0, D}$ and associated norms $\|\cdot\|_{0, D}$, respectively. Further, we denote by $H^{1}(D)$ the Sobolev space of square integrable functions with square integrable weak derivatives equipped with the inner product $(\cdot, \cdot)_{1, D}$ and norm $\|\cdot\|_{1, D}$. For $\Sigma \subseteq \partial D$, we refer to $H^{1 / 2}(\Sigma)$ as the space of traces $\left.v\right|_{\Sigma}$ of functions $v \in H^{1}(D)$ on $\Sigma$. We set $H_{0, \Sigma}^{1}(D):=\left\{v \in H^{1}(\Omega)|v|_{\Sigma}=0\right\}$ and refer to $H_{\Sigma}^{-1}(D)$ as the associated dual space. For a simply connected polyhedral domain $\Omega$ with boundary $\Gamma=\partial \Omega$ which can be split into $J$ relatively open faces $\Gamma_{1}, \ldots, \Gamma_{J}$ with $\Gamma=\cup_{j=1}^{J} \bar{\Gamma}_{j}$, we refer to $\mathbf{H}(\mathbf{c u r l} ; \Omega)$ as the Hilbert space $\mathbf{H}(\mathbf{c u r l} ; \Omega):=\left\{\mathbf{u} \in \mathbf{L}^{\mathbf{2}}(\Omega) \mid\right.$ curl $\left.\mathbf{u} \in \mathbf{L}^{\mathbf{2}}(\Omega)\right\}$, equipped with the inner product $(\mathbf{u}, \mathbf{v})_{c u r l, \Omega}:=(\mathbf{u}, \mathbf{v})_{0, \Omega}+(\mathbf{c u r l} \mathbf{u}, \mathbf{c u r l} \mathbf{v})_{0, \Omega}$ and the associated norm $\|\cdot\|_{c u r l, \Omega}$. We further refer to $\mathbf{H}\left(\mathbf{c u r l}^{\mathbf{0}} ; \Omega\right)$ as the subspace of irrotational vector fields. The space $\mathbf{H}(\operatorname{div} ; \Omega)$ is defined by $\mathbf{H}(\operatorname{div} ; \Omega):=\left\{\mathbf{q} \in \mathbf{L}^{2}(\Omega) \mid \operatorname{div} \mathbf{q} \in\right.$ $\left.L^{2}(\Omega)\right\}$ which is a Hilbert space with respect to the inner product $(\mathbf{u}, \mathbf{v})_{\operatorname{div}, \Omega}:=$ $(\mathbf{u}, \mathbf{v})_{0, \Omega}+(\operatorname{div} \mathbf{u}, \operatorname{div} \mathbf{v})_{0, \Omega}$ and the associated norm $\|\cdot\|_{\text {div }, \Omega}$. For vector fields $\mathbf{u} \in \mathbf{C}^{\infty}(\bar{\Omega})^{3}:=\left\{\left.\mathbf{u}\right|_{\Omega} \mid \mathbf{u} \in \mathbf{C}^{\infty}\left(\mathbb{R}^{3}\right)\right\}$, the normal component trace reads $\left.\eta_{\mathbf{n}}(\mathbf{u})\right|_{\Gamma_{j}}:=$ $\left.\mathbf{n}_{\Gamma_{j}} \cdot \mathbf{u}\right|_{\Gamma_{j}}, j=1, \ldots, J$ with the exterior unit normal vector $\mathbf{n}_{\Gamma_{j}}$ on $\Gamma_{j}$. The normal component trace mapping can be extended by continuity to a surjective, continuous linear mapping $\eta_{\mathbf{n}}: \mathbf{H}(\operatorname{div} ; \Omega) \rightarrow \mathbf{H}^{-1 / 2}(\Gamma)$ (cf. [32]; Thm. 2.2). We define $\mathbf{H}_{0}(\operatorname{div} ; \Omega)$ as the subspace of vector fields with vanishing normal components on 
$\Gamma$. In order to study the traces of vector fields $\mathbf{q} \in H(\mathbf{c u r l} ; \Omega)$, following [16], we introduce the spaces

$$
\begin{aligned}
\mathbf{L}_{\mathbf{t}}^{2}(\Gamma) & :=\left\{\mathbf{u} \in \mathbf{L}^{2}(\Omega) \mid \eta_{\mathbf{n}}(\mathbf{u})=0\right\}, \\
\mathbf{H}_{-}^{1 / 2}(\Gamma) & :=\left\{\mathbf{u} \in \mathbf{L}_{\mathbf{t}}^{2}(\Gamma)|\mathbf{u}|_{\Gamma_{j}} \in \mathbf{H}^{1 / 2}\left(\Gamma_{j}\right) \text { for all } j=1, \ldots, J\right\} .
\end{aligned}
$$

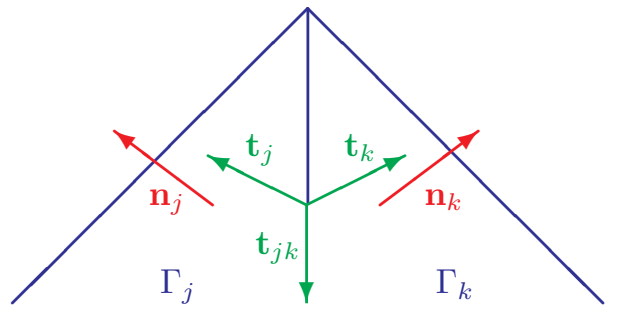

FIG. 2.1. Two adjacent faces $\Gamma_{j}, \Gamma_{k}$ with common edge $E_{j k}$

For $\Gamma_{j}, \Gamma_{k} \subset \Gamma$ with $j \neq k$ and $E_{j k}:=\bar{\Gamma}_{j} \cap \bar{\Gamma}_{k} \in \mathcal{E}_{h}$, the set of edges, we denote by $\mathbf{t}_{j}$ and $\mathbf{t}_{k}$ the tangential unit vectors along $\Gamma_{j}$ and $\Gamma_{k}$ and by $\mathbf{t}_{j k}$ the unit vector parallel to $E_{j k}$ such that $\Gamma_{j}$ is spanned by $\mathbf{t}_{j}, \mathbf{t}_{j k}$ and $\Gamma_{k}$ by $\mathbf{t}_{k}, \mathbf{t}_{j k}$ (cf. Figure 2.1). Let $\mathcal{J}:=\left\{(j, k) \in\{1, \ldots, J\}^{2} \mid \partial \Gamma_{j} \cap \partial \Gamma_{k}=E_{j k} \in \mathcal{E}_{h}\right\}$ and define

$$
\begin{aligned}
& \mathbf{H}_{\|}^{1 / 2}(\Gamma):=\left\{\mathbf{u} \in \mathbf{H}_{-}^{1 / 2}(\Gamma)\left|\left(\mathbf{t}_{j k} \cdot \mathbf{u}_{j}\right)\right|_{E_{j k}}=\left.\left(\mathbf{t}_{j k} \cdot \mathbf{u}_{k}\right)\right|_{E_{j k}} \text { for }(j, k) \in \mathcal{J}\right\}, \\
& \mathbf{H}_{\perp}^{1 / 2}(\Gamma):=\left\{\mathbf{u} \in \mathbf{H}_{-}^{1 / 2}(\Gamma)\left|\left(\mathbf{t}_{j} \cdot \mathbf{u}_{j}\right)\right|_{E_{j k}}=\left(\mathbf{t}_{k} \cdot \mathbf{u}_{k}\right)_{E_{j k}} \text { for }(j, k) \in \mathcal{J}\right\} .
\end{aligned}
$$

We refer to $\mathbf{H}_{\|}^{-1 / 2}(\Gamma)$ and $\mathbf{H}_{\perp}^{-1 / 2}(\Gamma)$ as the dual spaces of $\mathbf{H}_{\|}^{1 / 2}(\Gamma)$ and $\mathbf{H}_{\perp}^{1 / 2}(\Gamma)$ with $\mathbf{L}_{\mathbf{t}}^{2}(\Gamma)$ as the pivot space. For $\mathbf{u} \in \mathcal{D}(\bar{\Omega})^{3}$ we further define the tangential trace mapping $\left.\gamma_{\mathbf{t}}\right|_{\Gamma_{j}}:=\left.\mathbf{u} \wedge \mathbf{n}_{\Gamma_{j}}\right|_{\Gamma_{j}}, j=1, \ldots, J$, and the tangential components trace $\left.\boldsymbol{\pi}_{\mathbf{t}}\right|_{\Gamma_{j}}:=\left.\mathbf{n}_{\Gamma_{j}} \wedge\left(\mathbf{u} \wedge \mathbf{n}_{\Gamma_{j}}\right)\right|_{\Gamma_{j}}, j=1, \ldots, J$. Moreover, for a smooth function $u \in \mathcal{D}(\bar{\Omega})$ we define the tangential gradient operator $\nabla_{\Gamma}=\left.\operatorname{grad}\right|_{\Gamma}$ as the tangential components trace of the gradient operator $\nabla$, i.e., $\left.\nabla_{\Gamma} u\right|_{\Gamma_{j}}:=\nabla_{\Gamma_{j}} u=\pi_{\mathbf{t}, j}(\nabla u)=\mathbf{n}_{\Gamma_{j}} \wedge(\nabla u \wedge$ $\left.\mathbf{n}_{\Gamma_{j}}\right), j=1, \ldots, J$, which leads to a continuous linear mapping $\nabla_{\Gamma}: H^{3 / 2}(\Gamma) \rightarrow$ $\mathbf{H}_{\|}^{1 / 2}(\Gamma)$ (cf. [16]). The tangential divergence operator $\operatorname{div}_{\Gamma}: \mathbf{H}_{\|}^{-1 / 2}(\Gamma) \rightarrow H^{-3 / 2}(\Gamma)$ is defined, with the respective dual pairings $\langle\cdot, \cdot\rangle$, as the adjoint operator of $-\nabla_{\Gamma}$, i.e., $\left\langle\operatorname{div}_{\Gamma} \mathbf{u}, v\right\rangle=-\left\langle\mathbf{u}, \nabla_{\Gamma} v\right\rangle, v \in H^{3 / 2}(\Gamma), \mathbf{u} \in \mathbf{H}_{\|}^{-1 / 2}(\Gamma)$. Finally, for $u \in C^{\infty}(\bar{\Omega})$ we define the tangential curl operator $\left.\operatorname{curl}\right|_{\tau}$ as the tangential trace of the gradient operator

$$
\left.\operatorname{curl}_{\tau} u\right|_{\Gamma_{j}}=\operatorname{curl}_{\Gamma_{j}} u=\gamma_{\mathbf{t}, j}(\nabla u)=\nabla u \wedge \mathbf{n}_{j} \text { for } j=1, \ldots, J .
$$

The vectorial tangential curl operator is a linear continuous mapping

$$
\operatorname{curl}_{\tau}: H^{3 / 2}(\Gamma) \rightarrow \mathbf{H}_{\perp}^{1 / 2}(\Gamma) .
$$

The scalar tangential curl operator

$$
\operatorname{curl}_{\tau}: \mathbf{H}_{\perp}^{-1 / 2}(\Gamma) \rightarrow H^{-3 / 2}(\Gamma)
$$

is defined as the adjoint of the vectorial tangential curl operator via $\left.\operatorname{curl}\right|_{\tau}$, i.e.,

$$
\left\langle\left.\operatorname{curl}\right|_{\tau} \mathbf{u}, v>=\left\langle\mathbf{u},\left.\operatorname{curl}\right|_{\Gamma} v>\quad \text { for all } \quad v \in H^{3 / 2}(\Gamma) \text { and } \mathbf{u} \in \mathbf{H}_{\perp}^{-1 / 2}(\Gamma) .\right.\right.
$$


The range spaces of the tangential trace mapping $\gamma_{\mathbf{t}}$ and the tangential components trace mapping $\pi_{\mathbf{t}}$ on $H(\operatorname{curl} ; \Omega)$ can be characterized by means of the spaces

$$
\begin{aligned}
\mathbf{H}^{-1 / 2}\left(\left.\operatorname{div}\right|_{\Gamma}, \Gamma\right) & :=\left\{\boldsymbol{\lambda} \in \mathbf{H}_{\|}^{-1 / 2}(\Gamma)|\operatorname{div}|_{\Gamma} \boldsymbol{\lambda} \in H^{-1 / 2}(\Gamma)\right\}, \\
\mathbf{H}^{-1 / 2}\left(\left.\operatorname{curl}\right|_{\Gamma}, \Gamma\right) & :=\left\{\boldsymbol{\lambda} \in \mathbf{H}_{\perp}^{-1 / 2}(\Gamma)|\operatorname{curl}|_{\Gamma} \boldsymbol{\lambda} \in H^{-1 / 2}(\Gamma)\right\},
\end{aligned}
$$

which are dual to each other with respect to the pivot space $\mathbf{L}_{\mathbf{t}}^{2}(\Gamma)$. We refer to $\|\cdot\|_{-1 / 2, \text { div }_{\Gamma}, \Gamma}$ and $\|\cdot\|_{-1 / 2, \operatorname{curl}_{\Gamma}, \Gamma}$ as the respective norms and denote by $\langle\cdot, \cdot\rangle_{-1 / 2, \Gamma}$ the dual pairing (see, e.g., [16] for details).

It can be shown that the tangential trace mapping is a continuous linear mapping

$$
\gamma_{\mathbf{t}}: \mathbf{H}(\operatorname{curl} ; \Omega) \rightarrow \mathbf{H}^{-1 / 2}\left(\left.\operatorname{div}\right|_{\Gamma}, \Gamma\right),
$$

whereas the tangential components trace mapping is a continuous linear mapping

$$
\boldsymbol{\pi}_{\mathbf{t}}: \mathbf{H}(\operatorname{curl} ; \Omega) \rightarrow \mathbf{H}^{-1 / 2}\left(\left.\operatorname{curl}\right|_{\Gamma}, \Gamma\right) .
$$

The previous results imply that the tangential divergence of the tangential trace and the scalar tangential curl of the tangential components trace coincide: For $\mathbf{u} \in$ $\mathbf{H}(\mathbf{c u r l} ; \Omega)$ it holds $\operatorname{div}_{\Gamma}(\mathbf{u} \wedge \mathbf{n})=\left.\operatorname{curl}\right|_{\Gamma}(\mathbf{n} \wedge(\mathbf{u} \wedge \mathbf{n}))=\mathbf{n} \cdot \mathbf{c u r l} \mathbf{u}$. We define $\mathbf{H}_{\mathbf{0}}(\mathbf{c u r l} ; \Omega)$ as the subspace of $\mathbf{H}(\mathbf{c u r l} ; \Omega)$ with vanishing tangential traces on $\Gamma$.

Given a polyhedral domain $\Omega \subset \mathbb{R}^{3}$ with boundary $\Gamma=\partial \Omega$ such that $\Gamma=$ $\bar{\Gamma}_{D} \cup \bar{\Gamma}_{N}, \Gamma_{D} \cap \Gamma_{N}=\emptyset$, we denote by $\mathcal{T}_{H}(\Omega)$ a shape-regular simplicial triangulation of $\Omega$ that aligns with $\Gamma_{D}$ and $\Gamma_{N}$. We refer to $\mathcal{F}_{H}(\Omega)$ as the set of interior faces $F=T_{+} \cap T_{-}, T_{ \pm} \in \mathcal{T}_{H}(\Omega)$, and to $\mathcal{F}_{H}(\Sigma)$ as the set of faces located on the boundary $\Sigma \subseteq \Gamma$, while $\mathcal{F}_{H}(\bar{\Omega}):=\mathcal{F}_{H}(\Omega) \cup \mathcal{F}_{H}(\Gamma)$ is the set of all faces. Further, $\mathcal{E}_{H}(\Sigma)$ stands for the set of edges on $\Sigma$. We denote by $h_{T}$ and $h_{F}$ the diameter of an element $T \in \mathcal{T}_{H}(\Omega)$ and a face $F \in \mathcal{F}_{H}(\bar{\Omega})$, respectively. For two quantities $A, B \in \mathbb{R}_{+}$, we use the notation $A \lesssim B$, if there exists a constant $C \in \mathbb{R}_{+}$, independent of the mesh size of the triangulation $\mathcal{T}_{H}(\Omega)$, such that $A \leq C B$.

We refer to

$$
\mathbf{N d}^{\mathbf{1}}\left(\Omega ; \mathcal{T}_{H}(\Omega)\right):=\left\{\mathbf{v}_{H} \in \mathbf{H}(\operatorname{curl} ; \Omega)\left|\mathbf{v}_{H}\right|_{T} \in \mathbf{N D}^{\mathbf{1}}(T), T \in \mathcal{T}_{H}(\Omega)\right\}
$$

as the curl-conforming edge element space, where $\mathbf{N D}^{\mathbf{1}}(T)$ stands for the lowest order edge element of Nédélec's first family [43], and to

$$
\mathbf{N d}_{0, \Gamma_{D}}^{1}\left(\Omega ; \mathcal{T}_{H}(\Omega)\right):=\left\{\mathbf{v}_{H} \in \mathbf{N d}^{\mathbf{1}}\left(\Omega ; \mathcal{T}_{H}(\Omega)\right) \mid \gamma_{\mathbf{t}}\left(\mathbf{v}_{H}\right)=0 \text { on } \Gamma_{D}\right\}
$$

as its subspace of vanishing tangential trace components on $\Gamma_{D}$.

For vector fields $\mathbf{v}_{H} \in \prod_{T \in \mathcal{T}_{H}(\Omega)} \mathbf{H}(\mathbf{c u r l} ; T)$, we denote by $\|\cdot\|_{\text {curl }, H, \Omega}$ the meshdependent norm

$$
\left\|\mathbf{v}_{H}\right\|_{c u r l, H, \Omega}:=\left(\sum_{T \in \mathcal{T}_{H}(\Omega)}\left(\left\|\mathbf{v}_{H}\right\|_{0, T}^{2}+\left\|\operatorname{curl} \mathbf{v}_{H}\right\|_{0, T}^{2}\right)\right)^{1 / 2} .
$$

Moreover, for such vector fields we set $\left.\mathbf{v}_{H}^{ \pm}\right|_{F}:=\left.\left(\left.\mathbf{v}_{H}\right|_{T_{ \pm}}\right)\right|_{F}$ along $F=T_{+} \cap T_{-} \in \mathcal{F}_{H}(\Omega)$ and define

$$
\begin{aligned}
\left\{\mathbf{v}_{H}\right\} & :=\left\{\begin{array}{r}
\left(\mathbf{v}_{H}^{+}+\mathbf{v}_{H}^{-}\right) / 2, F \in \mathcal{F}_{H}(\Omega) \\
\mathbf{v}_{H}, F \in \mathcal{F}_{H}(\Gamma)
\end{array},\right. \\
{\left[\mathbf{v}_{H}\right]: } & =\left\{\begin{array}{r}
\mathbf{v}_{H}^{+}-\mathbf{v}_{H}^{-}, F \in \mathcal{F}_{H}(\Omega) \\
0, F \in \mathcal{F}_{H}(\Gamma)
\end{array}\right.
\end{aligned}
$$


as the averages and jumps of $\mathbf{v}_{H}$ across the interior faces $F$ of the triangulation. For scalar functions $v_{H} \in L^{2}(\Omega)$, the averages $\left\{v_{H}\right\}$ and jumps $\left[v_{H}\right]$ are defined analogously.

The class of $\mathbf{H}$ (curl)-elliptic boundary value problems to be approximated by IPDG-H methods is of the form

$$
\begin{aligned}
& \operatorname{curl} \mu^{-1} \operatorname{curl} \mathbf{u}+\sigma \mathbf{u}=\mathbf{f} \text { in } \Omega \text {, } \\
& \gamma_{\mathbf{t}}(\mathbf{u})=\mathbf{g}_{1} \text { on } \Gamma_{D}, \\
& \boldsymbol{\pi}_{\mathbf{t}}\left(\mu^{-1} \operatorname{curl} \mathbf{u}\right)=\mathbf{g}_{2} \text { on } \Gamma_{N} \text {. }
\end{aligned}
$$

We assume that $\mathbf{f} \in \mathbf{L}^{2}(\Omega), \mathbf{g}_{1} \in \mathbf{L}^{2}\left(\Gamma_{D}\right)$, and $\mathbf{g}_{2} \in \mathbf{H}\left(\operatorname{curl}_{\Gamma_{N}}^{0} ; \Gamma_{N}\right)$. We further suppose that $\mu$ is a symmetric, uniformly positive definite matrix-valued function $\mu=\mu(x), x \in \Omega$, and that $\sigma$ is a scalar nonnegative function $\sigma=\sigma(x), x \in \Omega$, that are elementwise constant with respect to a given coarse simplicial triangulation $\mathcal{T}_{H}(\Omega)$ of the computational domain.

We note that the subsequent analysis also applies to $\mathrm{H}(\mathrm{curl})$-elliptic problems in $2 \mathrm{D}$ as given by

$$
\begin{aligned}
\operatorname{curl} \mu^{-1} \operatorname{curl} \mathbf{u}+\sigma \mathbf{u} & =\mathbf{f} \quad \text { in } \Omega, \\
\mathbf{t}_{\Gamma_{D}} \cdot \mathbf{u} & =g_{1} \quad \text { on } \Gamma_{D}, \\
\mu^{-1} \operatorname{curl} \mathbf{u} & =g_{2} \quad \text { on } \Gamma_{N},
\end{aligned}
$$

where curl $\mathbf{u}=\partial u_{2} / \partial x_{1}-\partial u_{1} / \partial x_{2}$ for $\mathbf{u}=\left(u_{1}, u_{2}\right)^{T}$, whereas curl $u=\left(\partial u / \partial x_{2}\right.$, $\left.-\partial u / \partial x_{1}\right)^{T}$ for a scalar function $u$. Moreover, $\mathbf{t}_{\Gamma_{D}}$ stands for the tangential unit vector on the Dirichlet part $\Gamma_{D}$ of the boundary. The data $\mathbf{f}, g_{1}$ and $g_{2}$ have to be chosen accordingly.

We will develop the IPDG-H method and perform the a posteriori error analysis only in the $3 \mathrm{D}$ case. The necessary modifications for $2 \mathrm{D}$ problems are straightforward.

3. Hybridized IPDG Methods. A mixed formulation of (2.2a)-(2.2c) can be derived by introducing $\mathbf{p}:=\mu^{-1} \mathbf{c u r l} \mathbf{u}$ as an additional variable. Setting

$$
\begin{aligned}
\mathbf{V} & :=\left\{\mathbf{v} \in \mathbf{H}(\operatorname{curl} ; \Omega) \mid \gamma_{\mathbf{t}}(\mathbf{u})=\mathbf{g}_{1} \text { on } \Gamma_{D}\right\}, \mathbf{Q}:=\mathbf{L}^{2}(\Omega), \\
\mathbf{V}_{\mathbf{0}} & :=\left\{\mathbf{v} \in \mathbf{H}(\mathbf{c u r l} ; \Omega) \mid \gamma_{\mathbf{t}}(\mathbf{u})=\mathbf{0} \text { on } \Gamma_{D}\right\}
\end{aligned}
$$

it amounts to the computation of $(\mathbf{u}, \mathbf{p}) \in \mathbf{V} \times \mathbf{Q}$ with

$$
\begin{aligned}
a(\mathbf{p}, \mathbf{q})-b(\mathbf{u}, \mathbf{q})=\ell^{(1)}(\mathbf{q}) \quad \text { for all } \mathbf{q} \in \mathbf{Q} \\
b(\mathbf{v}, \mathbf{p})+c(\mathbf{u}, \mathbf{v})=\ell^{(2)}(\mathbf{v}) \quad \text { for all } \mathbf{v} \in \mathbf{V}_{\mathbf{0}}
\end{aligned}
$$


The bilinear forms $a, b$ and $c$ and the functionals $\ell^{(1)} \in \mathbf{Q}^{*}, \ell^{(2)} \in \mathbf{V}_{\mathbf{0}}^{*}$ are given by

$$
\begin{aligned}
& a(\mathbf{p}, \mathbf{q}):=\int_{\Omega} \mu \mathbf{p} \cdot \mathbf{q} d x \\
& b(\mathbf{u}, \mathbf{q}):=\int_{\Omega} \mathbf{c u r l} \mathbf{u} \cdot \mathbf{q} d x \\
& c(\mathbf{u}, \mathbf{v}):=\int_{\Omega} \sigma \mathbf{u} \cdot \mathbf{v} d x \\
& \ell^{(1)}(\mathbf{q}):=0 \\
& \ell^{(2)}(\mathbf{v}):=\int_{\Omega} \mathbf{f} \cdot \mathbf{v} d x+\int_{\Gamma_{N}} \mathbf{g}_{2} \cdot \gamma_{\mathbf{t}}(\mathbf{v}) d \tau .
\end{aligned}
$$

The operator-theoretic framework involves the operator $\mathcal{A}:(\mathbf{V} \times \mathbf{Q}) \rightarrow\left(\mathbf{V}_{\mathbf{0}} \times \mathbf{Q}\right)^{*}$ defined, for all $(\mathbf{u}, \mathbf{p}) \in \mathbf{V} \times \mathbf{Q}$ and all $(\mathbf{v}, \mathbf{q}) \in \mathbf{V}_{\mathbf{0}} \times \mathbf{Q}$ by

$$
(\mathcal{A}(\mathbf{u}, \mathbf{p}))(\mathbf{v}, \mathbf{q}):=a(\mathbf{p}, \mathbf{q})-b(\mathbf{u}, \mathbf{q})+b(\mathbf{v}, \mathbf{p})+c(\mathbf{u}, \mathbf{v}) .
$$

Then, the system (3.2a)-(3.2b) can be written in compact form as

$$
\mathcal{A}(\mathbf{u}, \mathbf{p})=\ell
$$

where $\ell(\mathbf{v}, \mathbf{q}):=\ell^{(1)}(\mathbf{q})+\ell^{(2)}(\mathbf{v})$ for all $(\mathbf{v}, \mathbf{q}) \in \mathbf{V}_{\mathbf{0}} \times \mathbf{Q}$.

ThEOREM 3.1. Under the assumptions on the data of (2.2a)-(2.2c), $\mathcal{A}$ is a continuous, bijective linear operator. Hence, for any $\left(\ell^{(1)}, \ell^{(2)}\right) \in \mathbf{Q}^{*} \times \mathbf{V}_{0}^{*}$, the system (3.2a)-(3.2b) admits a unique solution $(\mathbf{u}, \mathbf{p}) \in \mathbf{V} \times \mathbf{Q}$ which continuously depends on the data, namely

$$
\|(\mathbf{u}, \mathbf{p})\|_{\mathbf{V} \times \mathbf{Q}} \lesssim\left\|\ell^{(1)}\right\|_{Q^{*}}+\left\|\ell^{(2)}\right\|_{V_{0}^{*}}
$$

Proof. The mapping properties are straightforward. If $\mathbf{g}_{1} \neq \mathbf{0}$, there exists a unique $\mathbf{u}_{\mathbf{g}_{1}} \in \mathbf{V}$ such that for all $\mathbf{v} \in \mathbf{V}_{0}$ (cf., e.g., [42])

$$
\left(\mathcal{A}\left(\mathbf{u}_{\mathbf{g}_{1}}, \mathbf{0}\right)\right)\left(\mathbf{v},-\mu^{-1} \operatorname{curl} \mathbf{v}\right)=\int_{\Omega}\left(\mu^{-1} \operatorname{curl} \mathbf{u}_{\mathbf{g}_{1}} \cdot \operatorname{curl} \mathbf{v}+\sigma \mathbf{u}_{\mathbf{g}_{1}} \cdot \mathbf{v}\right) d x=0,
$$

and hence, we may restrict ourselves to the case of $\mathcal{A}: \mathbf{V}_{\mathbf{0}} \times \mathbf{Q} \rightarrow\left(\mathbf{V}_{\mathbf{0}} \times \mathbf{Q}\right)^{*}$. Now, for any $(\mathbf{u}, \mathbf{p}) \in \mathbf{V}_{\mathbf{0}} \times \mathbf{Q}$ we have

$$
\begin{aligned}
(\mathcal{A}(\mathbf{u}, \mathbf{p}))\left(3 \mathbf{u}, 2 \mathbf{p}-\mu^{-1} \mathbf{c u r l} \mathbf{u}\right) & =\left(\mathcal{A}\left(3 \mathbf{u}, 2 \mathbf{p}+\mu^{-1} \mathbf{c u r l} \mathbf{u}\right)\right)(\mathbf{u}, \mathbf{p}) \\
& =2 \mu\|\mathbf{p}\|_{L^{2}(\Omega)}^{2}+3 \sigma\|\mathbf{u}\|_{L^{2}(\Omega)}^{2}+\mu^{-1}\|\mathbf{c u r l} \mathbf{u}\|_{L^{2}(\Omega)}^{2}
\end{aligned}
$$

This implies the inf-sup condition and the remaining degeneracy condition which implies bijectivity.

Given a simplicial triangulation $\mathcal{T}_{H}(\Omega)$, DG methods are based on the approximation of the vector field $\mathbf{u}$ and $\mathbf{p}$ by elementwise polynomials, thus giving rise to the 
finite dimensional function spaces

$$
\begin{array}{r}
\mathbf{V}_{H}:=\left\{\mathbf{v}_{H} \in \mathbf{L}^{2}(\Omega)\left|\mathbf{v}_{H}\right|_{T} \in\left(\mathbf{\Pi}_{k}(T)\right), T \in \mathcal{T}_{H}(\Omega),\right. \\
\left.\gamma_{\mathbf{t}}\left(\mathbf{v}_{H}\right)=\mathbf{g}_{H, 1} \text { on } F \in \mathcal{F}_{H}\left(\Gamma_{D}\right)\right\}, \\
\mathbf{Q}_{H}:=\left\{\mathbf{q}_{H} \in \mathbf{L}^{\mathbf{2}}(\Omega)\left|\mathbf{q}_{H}\right|_{T} \in \mathbf{\Pi}_{k}(T), T \in \mathcal{T}_{H}(\Omega)\right\} .
\end{array}
$$

Here and in the sequel, $\mathbf{g}_{H, 1} \in \boldsymbol{\Pi}_{k}(F), F \in \mathcal{F}_{H}\left(\Gamma_{D}\right)$ is some approximation of $\mathbf{g}_{1}$ and $\boldsymbol{\Pi}_{k}(T), T \in \mathcal{T}_{H}(\Omega)$, as well as $\boldsymbol{\Pi}_{k}(F), F \in \mathcal{F}_{H}(\bar{\Omega})$, stand for the sets of vector-valued functions whose components are polynomials of degree at most $k \in \mathbb{N}$.

DG methods amount to the computation of $\left(\mathbf{p}_{H}, \mathbf{u}_{H}\right) \in \mathbf{Q}_{H} \times \mathbf{V}_{H}$ with

$$
\begin{array}{r}
a_{H}\left(\mathbf{p}_{H}, \mathbf{q}_{H}\right)-b_{H}\left(\mathbf{u}_{H}, \mathbf{q}_{H}\right)+d_{H}\left(\hat{\mathbf{u}}_{H}, \mathbf{q}_{H}\right)=\ell_{H}^{(1)}\left(\mathbf{q}_{H}\right) \text { for all } \mathbf{q}_{H} \in \mathbf{Q}_{H}, \\
b_{H}\left(\mathbf{v}_{H}, \mathbf{p}_{H}\right)-d_{H}\left(\mathbf{v}_{H}, \hat{\mathbf{p}}_{H}\right)+c_{H}\left(\mathbf{u}_{H}, \mathbf{v}_{H}\right)=\ell_{H}^{(2)}\left(\mathbf{v}_{H}\right) \text { for all } \mathbf{v}_{H} \in \mathbf{V}_{H} .
\end{array}
$$

Here and throughout, $\hat{\mathbf{u}}_{H}, \hat{\mathbf{p}}_{H}$ are appropriate numerical flux functions and the meshdependent bilinear forms $a_{H}, b_{H}, c_{H}$, and $d_{H}$ are defined by means of

$$
\begin{aligned}
a_{H}\left(\mathbf{p}_{H}, \mathbf{q}_{H}\right) & :=\sum_{T \in \mathcal{T}_{H}(\Omega)} \int_{T} \mu \mathbf{p}_{H} \cdot \mathbf{q}_{H} d x, \\
b_{H}\left(\mathbf{u}_{H}, \mathbf{q}_{H}\right) & :=\sum_{T \in \mathcal{T}_{H}(\Omega)} \int_{T} \operatorname{curl} \mathbf{u}_{H} \cdot \mathbf{q}_{H} d x, \\
c_{H}\left(\mathbf{u}_{H}, \mathbf{v}_{H}\right) & :=\sum_{T \in \mathcal{T}_{H}(\Omega)} \int_{T} \sigma \mathbf{u}_{H} \cdot \mathbf{v}_{H} d x, \\
d_{H}\left(\mathbf{u}_{H}, \mathbf{q}_{H}\right) & :=\sum_{F \in \mathcal{F}_{H}(\bar{\Omega})}\left\langle\gamma_{\mathbf{t}}\left(\mathbf{u}_{H}\right), \boldsymbol{\pi}_{\mathbf{t}}\left(\mathbf{q}_{H}\right)\right\rangle .
\end{aligned}
$$

The functionals $\ell_{H}^{(1)}$ and $\ell_{H}^{(2)}$ are given by

$$
\begin{aligned}
\ell_{H}^{(1)}\left(\mathbf{q}_{H}\right) & :=0, \\
\ell_{H}^{(2)}\left(\mathbf{v}_{H}\right) & :=\sum_{T \in \mathcal{T}_{H}(\Omega)} \int_{T} \mathbf{f} \cdot \mathbf{v}_{H} d x+\sum_{F \in \mathcal{F}_{H}\left(\Gamma_{N}\right)} \int_{F} \mathbf{g}_{2} \cdot \gamma_{\mathbf{t}}\left(\mathbf{v}_{H}\right) d \tau .
\end{aligned}
$$

In case of symmetric Interior Penalty Discontinuous Galerkin (IPDG) methods, the numerical fluxes read

$$
\begin{aligned}
& \boldsymbol{\gamma}_{\mathbf{t}}\left(\hat{\mathbf{u}}_{\mathbf{H}}\right):=\left\{\begin{array}{r}
\left\{\boldsymbol{\gamma}_{\mathbf{t}}\left(\mathbf{u}_{H}\right)\right\}, F \in \mathcal{F}_{H}(\Omega) \\
0, F \in \mathcal{F}_{H}(\Gamma)
\end{array}\right. \\
& \boldsymbol{\pi}_{\mathbf{t}}\left(\hat{\mathbf{p}}_{\mathbf{H}}\right):=\left\{\begin{array}{r}
\left\{\boldsymbol{\pi}_{\mathbf{t}}\left(\mu^{-1} \mathbf{c u r l} \mathbf{u}_{H}\right)\right\}-\alpha h_{F}^{-1}\left[\boldsymbol{\gamma}_{\mathbf{t}}\left(\mathbf{u}_{H}\right)\right], F \in \mathcal{F}_{H}(\Omega) \\
0, F \in \mathcal{F}_{H}(\Gamma)
\end{array}\right.
\end{aligned}
$$

with a suitable penalty parameter $\alpha>0$. The choice $\mathbf{q}_{H}:=\mu^{-1} \operatorname{curl} \mathbf{v}_{H}$ in (3.8a) and (3.11a),(3.11b) allow the elimination of $\mathbf{p}_{H}$ from (3.8a),(3.8b). This results in the following standard form of the symmetric IPDG method: Find $\mathbf{u}_{H} \in \mathbf{V}_{H}$ such that

$$
a_{I P}\left(\mathbf{u}_{H}, \mathbf{v}_{H}\right)=\ell_{I P}\left(\mathbf{v}_{H}\right) \text { for all } \mathbf{v}_{H} \in \mathbf{V}_{H} \text {. }
$$


Here and in the sequel, the bilinear form $a_{I P}$ and the functional $\ell_{I P}$ read

$$
\begin{aligned}
& a_{I P}\left(\mathbf{u}_{H}, \mathbf{v}_{H}\right):=\sum_{T \in \mathcal{T}_{H}(\Omega)} \int_{T}\left(\mu^{-1} \mathbf{c u r l} \mathbf{u}_{H} \cdot \operatorname{curl} \mathbf{v}_{H}+\sigma \mathbf{u}_{H} \cdot \mathbf{v}_{H}\right) d x \\
& -\sum_{F \in \mathcal{F}_{H}(\Omega)} \int_{F}\left(\left\{\boldsymbol{\pi}_{\mathbf{t}}\left(\mu^{-1} \mathbf{c u r l} \mathbf{u}_{H}\right)\right\} \cdot\left[\boldsymbol{\gamma}_{\mathbf{t}}\left(\mathbf{v}_{H}\right)\right]+\left\{\boldsymbol{\gamma}_{\mathbf{t}}\left(\mathbf{u}_{H}\right)\right\} \cdot\left[\boldsymbol{\pi}_{\mathbf{t}}\left(\mu^{-1} \mathbf{c u r l} \mathbf{v}_{H}\right)\right]\right) d \tau \\
& +\alpha \sum_{F \in \mathcal{F}_{H}(\Omega)} h_{F}^{-1} \int_{F}\left[\gamma_{\mathbf{t}}\left(\mathbf{u}_{H}\right)\right] \cdot\left[\gamma_{\mathbf{t}}\left(\mathbf{v}_{H}\right)\right] d \tau, \\
& \ell_{I P}\left(\mathbf{v}_{H}\right):=\sum_{T \in \mathcal{T}_{H}(\Omega)} \int_{T} \mathbf{f} \cdot \mathbf{v}_{H} d x+\sum_{F \in \mathcal{F}_{H}\left(\Gamma_{N}\right)} \int_{F} \mathbf{g}_{2} \cdot \boldsymbol{\gamma}_{\mathbf{t}}\left(\mathbf{v}_{H}\right) d \tau
\end{aligned}
$$

The idea of hybridization is to enforce the continuity of the tangential component traces of $\mathbf{p}_{H}$ across the interior edges of the triangulation by a piecewise polynomial Lagrange multiplier which is an approximation of the tangential traces of $\mathbf{u}$. For this purpose, we introduce the multiplier space

$$
\mathbf{M}_{H}:=\left\{\boldsymbol{\mu}_{H} \in \mathbf{L}^{\mathbf{2}}\left(\mathcal{F}_{H}(\bar{\Omega})\right)\left|\boldsymbol{\mu}_{H}\right|_{F} \in \boldsymbol{\Pi}_{k}(F), F \in \mathcal{F}_{H}(\bar{\Omega})\right\} .
$$

Choosing a numerical flux function $\hat{\mathbf{p}}_{H}$, not necessarily the same as in $(3.11 \mathrm{~b})$, the IPDG-H method is to find $\left(\mathbf{p}_{H}, \mathbf{u}_{H}, \boldsymbol{\lambda}_{H}\right) \in \mathbf{Q}_{H} \times \mathbf{V}_{H} \times \mathbf{M}_{H}$ with

$$
\begin{aligned}
a_{H}\left(\mathbf{p}_{H}, \mathbf{q}_{h}\right)-b_{H}\left(\mathbf{u}_{H}, \mathbf{q}_{H}\right)+d_{H}\left(\boldsymbol{\lambda}_{H}, \mathbf{q}_{H}\right) & =\ell_{H}^{(1)}\left(\mathbf{q}_{H}\right) \text { for all } \mathbf{q}_{H} \in \mathbf{Q}_{H} \\
b_{H}\left(\mathbf{v}_{H}, \mathbf{p}_{H}\right)-d_{H}\left(\mathbf{v}_{H}, \hat{\mathbf{p}}_{H}\right)+c_{H}\left(\mathbf{u}_{H}, \mathbf{v}_{H}\right) & =\ell_{H}^{(2)}\left(\mathbf{v}_{H}\right) \text { for all } \mathbf{v}_{H} \in \mathbf{V}_{H} \\
d_{H}\left(\boldsymbol{\mu}_{H}, \hat{\mathbf{p}}_{H}\right) & =0 \quad \text { for all } \boldsymbol{\mu}_{H} \in \mathbf{M}_{H} .
\end{aligned}
$$

In IPDG-H methods, the penalty parameter $\alpha$ is typically chosen elementwise, i.e., $\left.\alpha\right|_{T}=\alpha_{T}, T \in \mathcal{T}_{H}(\Omega)$, so that on $F \in \mathcal{F}_{H}(\Omega)$ with $F=T_{+} \cap T_{-}, T_{ \pm} \in \mathcal{T}_{H}(\Omega)$, we have to distinguish between $\alpha_{+}:=\alpha_{T_{+}}$and $\alpha_{-}:=\alpha_{T_{-}}$.

The advantage of hybridized methods is that the primal and dual variables $\mathbf{u}_{H}$ and $\mathbf{p}_{H}$ can be eliminated from $(3.15 \mathrm{a})-(3.15 \mathrm{c})$ which results in a global variational problem for the Lagrange multiplier $\boldsymbol{\lambda}_{H} \in \mathbf{M}_{H}$ of the form

$$
a_{H}^{(S)}\left(\boldsymbol{\lambda}_{H}, \boldsymbol{\mu}_{H}\right)=\ell_{H}^{(S)}\left(\boldsymbol{\mu}_{H}\right) \quad \text { for all } \boldsymbol{\mu}_{H} \in \mathbf{M}_{H}
$$

Once $\boldsymbol{\lambda}_{H} \in \mathbf{M}_{H}$ has been computed, the primal and dual variables can be computed by the solution of low-dimensional, local problems. To this end, following the unified framework from [28], we set

$$
\begin{gathered}
\boldsymbol{\lambda}_{H}=\left\{\begin{array}{r}
\mathbf{u}_{H} \text { on } \partial T \backslash \Gamma_{D} \\
0 \text { on } \partial T \cap \Gamma_{D}
\end{array}, \quad \overline{\mathbf{g}}_{H, 1}=\left\{\begin{array}{r}
0 \text { on } \partial T \backslash \Gamma_{D} \\
\mathbf{g}_{H, 1} \text { on } \partial T \cap \Gamma_{D}
\end{array},\right.\right. \\
\overline{\mathbf{g}}_{H, 2}=\left\{\begin{array}{r}
0 \text { on } \partial T \backslash \Gamma_{N} \\
\mathbf{g}_{H, 2} \text { on } \partial T \cap \Gamma_{N}
\end{array}\right.
\end{gathered}
$$

with an approximation $\mathbf{g}_{H, 2} \in \boldsymbol{\Pi}_{k}(F), F \in \mathcal{F}_{H}\left(\Gamma_{N}\right)$, of $\mathbf{g}_{2}$. We define

$$
\begin{aligned}
&\left(\mathbf{S}_{\mathbf{p}} \mathbf{f}, \mathbf{S}_{\mathbf{u}} \mathbf{f}\right) \in \mathbf{\Pi}_{k}(T)^{2}, \quad\left(\mathbf{S}_{\mathbf{p}} \boldsymbol{\lambda}_{H}, \mathbf{S}_{\mathbf{u}} \boldsymbol{\lambda}_{H}\right) \in \mathbf{\Pi}_{k}(T)^{2}, \\
&\left(\mathbf{S}_{\mathbf{p}} \mathbf{g}_{H, 1}, \mathbf{S}_{\mathbf{u}} \mathbf{g}_{H, 1}\right) \in \mathbf{\Pi}_{k}(T)^{2} \quad, \quad\left(\mathbf{S}_{\mathbf{p}} \mathbf{g}_{H, 2}, \mathbf{S}_{\mathbf{u}} \mathbf{g}_{H, 2}\right) \in \mathbf{\Pi}_{k}(T)^{2}
\end{aligned}
$$


as the solutions of the local problems

$$
\begin{aligned}
\mu \mathbf{S}_{\mathbf{p}} \mathbf{f}-\operatorname{curl} \mathbf{S}_{\mathbf{u}} \mathbf{f} & =0 \quad \text { in } T, \\
\mathbf{c u r l} \mathbf{S}_{\mathbf{p}} \mathbf{f}+\sigma \mathbf{S}_{\mathbf{u}} \mathbf{f} & =\mathbf{f} \quad \text { in } T, \\
\gamma_{\mathbf{t}}\left(\mathbf{S}_{\mathbf{u}} \mathbf{f}\right) & =0 \quad \text { on } \partial T, \\
\mu \mathbf{S}_{\mathbf{p}} \boldsymbol{\lambda}_{H}-\operatorname{curl} \mathbf{S}_{\mathbf{u}} \boldsymbol{\lambda}_{H} & =0 \quad \text { in } T, \\
\mathbf{c u r l} \mathbf{S}_{\mathbf{p}} \boldsymbol{\lambda}_{H}+\sigma \mathbf{S}_{\mathbf{u}} \boldsymbol{\lambda}_{H} & =0 \quad \text { in } T, \\
\boldsymbol{\gamma}_{\mathbf{t}}\left(\mathbf{S}_{\mathbf{u}} \boldsymbol{\lambda}_{H}\right) & =\boldsymbol{\lambda}_{H} \quad \text { on } \partial T, \\
\mu \mathbf{S}_{\mathbf{p}} \overline{\mathbf{g}}_{H, 1}-\operatorname{curl} \mathbf{S}_{\mathbf{u}} \overline{\mathbf{g}}_{H, 1} & =0 \quad \text { in } T, \\
\mathbf{c u r l} \mathbf{S}_{\mathbf{p}} \overline{\mathbf{g}}_{H, 1}+\sigma \mathbf{S}_{\mathbf{u}} \overline{\mathbf{g}}_{H, 1} & =0 \quad \text { in } T, \\
\boldsymbol{\gamma}_{\mathbf{t}}\left(\mathbf{S}_{\mathbf{u}} \overline{\mathbf{g}}_{H, 1}\right) & =\overline{\mathbf{g}}_{H, 1} \quad \text { on } \partial T, \\
\mu \mathbf{S}_{\mathbf{p}} \overline{\mathbf{g}}_{H, 2}-\operatorname{curl} \mathbf{S}_{\mathbf{u}} \overline{\mathbf{g}}_{H, 2} & =0 \quad \text { in } T, \\
\mathbf{c u r l} \mathbf{S}_{\mathbf{p}} \overline{\mathbf{g}}_{H, 2}+\sigma \mathbf{S}_{\mathbf{u}} \overline{\mathbf{g}}_{H, 2} & =0 \quad \text { in } T, \\
\boldsymbol{\pi}_{\mathbf{t}}\left(\mathbf{S}_{\mathbf{u}} \overline{\mathbf{g}}_{H, 2}\right) & =\overline{\mathbf{g}}_{H, 2} \quad \text { on } \partial T .
\end{aligned}
$$

The numerical flux $\boldsymbol{\pi}_{\mathbf{t}}\left(\hat{\mathbf{p}}_{H}\right)$ is given by means of local numerical fluxes

$$
\boldsymbol{\pi}_{\mathbf{t}}\left(\hat{\mathbf{p}}_{H}\right)=\hat{\mathbf{S}}_{\mathbf{p}} \mathbf{f}+\hat{\mathbf{S}}_{\mathbf{p}} \boldsymbol{\lambda}_{H}+\hat{\mathbf{S}}_{\mathbf{p}} \overline{\mathbf{g}}_{H, 1}+\hat{\mathbf{S}}_{\mathbf{p}} \overline{\mathbf{g}}_{H, 2}
$$

In particular, for the IPDG-H method (3.15a)-(3.15c) we choose

$$
\begin{aligned}
& \hat{\mathbf{S}}_{\mathbf{p}} \mathbf{f}=\left\{\begin{array}{l}
\boldsymbol{\pi}_{\mathbf{t}}\left(\mu^{-1} \operatorname{curl} \mathbf{S}_{\mathbf{u}} \mathbf{f}\right)-\alpha_{T} h_{F}^{-1} \gamma_{\mathbf{t}}\left(\mathbf{S}_{\mathbf{u}} \mathbf{f}\right) \text { on } F \in \mathcal{F}_{H}\left(\Omega \cup \Gamma_{D}\right), \\
\boldsymbol{\pi}_{\mathbf{t}}\left(\mu^{-1} \operatorname{curl} \mathbf{S}_{\mathbf{u}} \mathbf{f}\right)-\alpha_{T} h_{F}^{-1} \boldsymbol{\pi}_{\mathbf{t}}\left(\mu^{-1} \mathbf{c u r l} \mathbf{S}_{\mathbf{u}} \mathbf{f}\right) \text { on } F \in \mathcal{F}_{H}\left(\Gamma_{N}\right),
\end{array}\right. \\
& \hat{\mathbf{S}}_{\mathbf{p}} \boldsymbol{\lambda}_{H}=\left\{\begin{array}{l}
\boldsymbol{\pi}_{\mathbf{t}}\left(\mu^{-1} \operatorname{curl} \mathbf{S}_{\mathbf{u}} \boldsymbol{\lambda}_{H}\right) \\
-\alpha_{T} h_{F}^{-1}\left(\boldsymbol{\gamma}_{\mathbf{t}}\left(\mathbf{S}_{\mathbf{u}} \boldsymbol{\lambda}_{H}\right)-\boldsymbol{\lambda}_{H}\right) \text { on } F \in \mathcal{F}_{H}\left(\Omega \cup \Gamma_{D}\right), \\
\boldsymbol{\pi}_{\mathbf{t}}\left(\mu^{-1} \operatorname{curl} \mathbf{S}_{\mathbf{u}} \boldsymbol{\lambda}_{H}\right) \\
-\alpha_{T} h_{F}^{-1}\left(\boldsymbol{\pi}_{\mathbf{t}}\left(\mu^{-1} \operatorname{curl} \mathbf{S}_{\mathbf{u}} \boldsymbol{\lambda}_{\mathbf{H}}\right)-\boldsymbol{\lambda}_{H}\right) \text { on } F \in \mathcal{F}_{H}\left(\Gamma_{N}\right),
\end{array}\right. \\
& \hat{\mathbf{S}}_{\mathbf{p}} \overline{\mathbf{g}}_{H, i}=\left\{\begin{array}{l}
\boldsymbol{\pi}_{\mathbf{t}}\left(\mu^{-1} \operatorname{curl} \mathbf{S}_{\mathbf{u}} \overline{\mathbf{g}}_{H, i}\right) \\
-\alpha_{T} h_{F}^{-1}\left(\gamma_{\mathbf{t}}\left(\mathbf{S}_{\mathbf{u}} \overline{\mathbf{g}}_{H, i}\right)-\overline{\mathbf{g}}_{H, i}\right) \text { on } F \in \mathcal{F}_{H}\left(\Omega \cup \Gamma_{D}\right), \\
\boldsymbol{\pi}_{\mathbf{t}}\left(\mu^{-1} \mathbf{c u r l} \mathbf{S}_{\mathbf{u}} \overline{\mathbf{g}}_{H, i}\right) \\
-\alpha_{T} h_{F}^{-1}\left(\boldsymbol{\pi}_{\mathbf{t}}\left(\mu^{-1} \mathbf{c u r l} \mathbf{S}_{\mathbf{u}} \overline{\mathbf{g}}_{\mathbf{H}, \mathbf{i}}\right)-\overline{\mathbf{g}}_{H, i}\right) \text { on } F \in \mathcal{F}_{H}\left(\Gamma_{N}\right) .
\end{array}\right.
\end{aligned}
$$

For sufficiently large $\alpha_{T}, T \in \mathcal{T}_{H}(\bar{\Omega})$, both the local problems $(3.17 \mathrm{a})-(3.17 \mathrm{~d})$ and the global variational problem (3.16) have unique solutions which can be shown along the same lines of proof as in [28] for standard second order elliptic boundary value problems. If $\boldsymbol{\lambda}_{H} \in \mathbf{M}_{H}$ solves (3.16), then

$$
\begin{aligned}
& \mathbf{p}_{H}=\mathbf{S}_{\mathbf{p}} \mathbf{f}+\mathbf{S}_{\mathbf{p}} \boldsymbol{\lambda}_{H}+\mathbf{S}_{\mathbf{p}} \overline{\mathbf{g}}_{H, 1}+\mathbf{S}_{\mathbf{p}} \overline{\mathbf{g}}_{H, 2}, \\
& \mathbf{u}_{H}=\mathbf{S}_{\mathbf{u}} \mathbf{f}+\mathbf{S}_{\mathbf{u}} \boldsymbol{\lambda}_{H}+\mathbf{S}_{\mathbf{u}} \overline{\mathbf{g}}_{H, 1}+\mathbf{S}_{\mathbf{u}} \overline{\mathbf{g}}_{H, 2}
\end{aligned}
$$

defines the solution of (3.15a)-(3.15c).

TheOREM 3.2. Assume that the numerical flux $\hat{\mathbf{p}}_{H}$ is given by (3.18) and that $\left(\mathbf{p}_{H}, \mathbf{u}_{H}, \boldsymbol{\lambda}_{H}\right)$ is the solution of (3.15a)-(3.15c). Then, the numerical flux $\hat{\mathbf{p}}_{H}$ and the 
multiplier $\boldsymbol{\lambda}_{H}$ satisfy

$$
\begin{aligned}
\boldsymbol{\pi}_{\mathbf{t}}\left(\hat{\mathbf{p}}_{H}\right):=\left\{\begin{array}{l}
\bar{\alpha}^{-1}\left(\alpha_{-} \boldsymbol{\pi}_{\mathbf{t}}\left(\mu_{+}^{-1} \mathbf{c u r l} \mathbf{u}_{H}^{+}\right)+\alpha_{+} \boldsymbol{\pi}_{\mathbf{t}}\left(\mu_{-}^{-1} \text { curl } \mathbf{u}_{H}^{-}\right)\right. \\
\left.-\alpha_{+} \alpha_{-} h_{F}^{-1}\left[\gamma_{\mathbf{t}}\left(\mathbf{u}_{H}\right)\right]\right) \text { on } F \in \mathcal{F}_{H}(\Omega), \\
0 \text { on } F \in \mathcal{F}_{H}\left(\Gamma_{D}\right), \\
0 \text { on } F \in \mathcal{F}_{H}\left(\Gamma_{N}\right),
\end{array}\right. \\
\boldsymbol{\lambda}_{\mathbf{H}}=\left\{\begin{array}{c}
\bar{\alpha}^{-1}\left(\alpha_{+} \boldsymbol{\gamma}_{\mathbf{t}}\left(\mathbf{u}_{H}^{+}\right)+\alpha_{-} \gamma_{\mathbf{t}}\left(\mathbf{u}_{H}^{-}\right)\right. \\
\left.-h_{F}\left[\boldsymbol{\pi}_{\mathbf{t}}\left(\mu^{-1} \operatorname{curl} \mathbf{u}_{H}\right)\right]\right) \text { on } F \in \mathcal{F}_{H}(\Omega), \\
-\alpha_{T}^{-1} h_{F} \boldsymbol{\pi}_{\mathbf{t}}\left(\mu^{-1} \operatorname{curl} \mathbf{u}_{H}\right) \text { on } F \in \mathcal{F}_{H}\left(\Gamma_{D}\right), \\
-\alpha_{T}^{-1} h_{F} \boldsymbol{\pi}_{\mathbf{t}}\left(\mu^{-1} \operatorname{curl} \mathbf{u}_{H}\right) \text { on } F \in \mathcal{F}_{H}\left(\Gamma_{N}\right),
\end{array}\right.
\end{aligned}
$$

where $\bar{\alpha}:=\alpha_{+}+\alpha_{-}$on $F=\partial T_{+} \cap \partial T_{-}$for $T_{ \pm} \in \mathcal{T}_{H}(\Omega)$.

Proof. Let $F \in \mathcal{F}_{H}(\Omega)$. If we use (3.19a)-(3.19c) and (3.20) in (3.18), we obtain

$$
\boldsymbol{\pi}_{\mathbf{t}}\left(\hat{\mathbf{p}}_{H}\right)=\boldsymbol{\pi}_{\mathbf{t}}\left(\mu^{-1} \mathbf{c u r l} \mathbf{u}_{H}\right)-\alpha_{T} h_{F}^{-1}\left(\gamma_{\mathbf{t}}\left(\mathbf{u}_{H}\right)-\gamma_{\mathbf{t}}\left(\mathbf{S}_{\mathbf{u}} \boldsymbol{\lambda}_{H}\right)\right) \quad \text { on } F \text {. }
$$

Hence, observing (3.17b), it follows that

$$
\begin{aligned}
{\left[\boldsymbol{\pi}_{\mathbf{t}}\left(\hat{\mathbf{p}}_{H}\right)\right]=} & {\left[\boldsymbol{\pi}_{\mathbf{t}}\left(\mu^{-1} \operatorname{curl} \mathbf{u}_{H}\right)\right]-\left(\alpha_{+} h_{F}^{-1} \boldsymbol{\gamma}_{\mathbf{t}}\left(\mathbf{u}_{H}^{+}\right)\right.} \\
& \left.+\alpha_{-} h_{F}^{-1} \boldsymbol{\gamma}_{\mathbf{t}}\left(\mathbf{u}_{H}^{-}\right)\right)+\left(\alpha_{+}+\alpha_{-}\right) h_{F}^{-1} \boldsymbol{\lambda}_{H} .
\end{aligned}
$$

The specification (3.14) of the multiplier space $\mathbf{M}_{H}$ and equation (3.15c) imply $\left[\boldsymbol{\pi}_{\mathbf{t}}\left(\hat{\mathbf{p}}_{H}\right)\right]=0$. This results in (3.21b) due to (3.22). On the other hand,

$$
\boldsymbol{\pi}_{\mathbf{t}}\left(\hat{\mathbf{p}}_{H}^{ \pm}\right)=\boldsymbol{\pi}_{\mathbf{t}}\left(\mathbf{p}_{H}^{ \pm}\right)-\alpha_{ \pm} h_{F}^{-1}\left(\gamma_{\mathbf{t}}\left(\mathbf{u}_{H}^{ \pm}\right)-\boldsymbol{\lambda}_{H}\right) .
$$

We deduce (3.21a) by inserting (3.21b) into (3.23). The proof of (3.21a),(3.21b) for $F \in \mathcal{F}_{H}\left(\Gamma_{D}\right)$ and $F \in \mathcal{F}_{H}\left(\Gamma_{N}\right)$ follows from similar arguments.

The representation (3.21b) of the Lagrange multiplier $\boldsymbol{\lambda}_{\mathbf{H}}$ shows that it provides an approximation of the tangential trace on the interfaces $F \in \mathcal{F}_{H}(\Omega)$ which reminds of mortar methods for $\mathrm{H}$ (curl)-elliptic problems (cf., e.g., [20, 51]). Indeed, the IPDG$\mathrm{H}$ method (3.15a)-(3.15c) can be equivalently formulated as a mortar method. To see this, choose $\mathbf{q}_{H}=\mu^{-1}$ curl $\mathbf{u}_{H}$ in (3.15a) and the numerical flux $\hat{\mathbf{p}}_{H}$ in (3.15a) according to (3.21b). Then, by elimination of $\mathbf{p}_{H}$,

$$
\tilde{\boldsymbol{\lambda}}_{H}:=\boldsymbol{\lambda}_{H}-\bar{\alpha}^{-1}\left(\alpha_{+} \boldsymbol{\gamma}_{\mathbf{t}}\left(\mathbf{u}_{H}^{+}\right)+\alpha_{-} \boldsymbol{\gamma}_{\mathbf{t}}\left(\mathbf{u}_{H}^{-}\right)\right)
$$

satisfies

$$
\begin{aligned}
\tilde{a}_{H}\left(\mathbf{u}_{H}, \mathbf{v}_{H}\right)+\tilde{b}_{H}\left(\tilde{\lambda}_{H}, \mathbf{v}_{H}\right) & =\ell_{H}^{(2)}\left(\mathbf{v}_{H}\right) & & \text { for all } \mathbf{v}_{H} \in \mathbf{V}_{H}, \\
\tilde{b}_{H}\left(\boldsymbol{\mu}_{H}, \mathbf{u}_{H}\right)-\tilde{d}_{H}\left(\tilde{\boldsymbol{\lambda}}_{H}, \boldsymbol{\mu}_{H}\right) & =0 & & \text { for all } \boldsymbol{\mu}_{H} \in \mathbf{M}_{H} .
\end{aligned}
$$


Here and throughout the following, the bilinear forms $\tilde{a}_{H}, \tilde{b}_{H}$ and $\tilde{d}_{H}$ read

$$
\begin{aligned}
& \tilde{a}_{H}\left(\mathbf{u}_{H}, \mathbf{v}_{H}\right):=\sum_{T \in \mathcal{T}_{H}(\Omega)} \int_{T}\left(\mu^{-1} \operatorname{curl} \mathbf{u}_{H} \cdot \operatorname{curl} \mathbf{v}_{H}+\sigma \mathbf{u}_{H} \cdot \mathbf{v}_{H}\right) d x \\
& -\sum_{T \in \mathcal{T}_{H}(\Omega)} \int_{\partial T \cap \Omega} \bar{\alpha}^{-1} \alpha_{T} \boldsymbol{\pi}_{\mathbf{t}}\left(\mu^{-1} \operatorname{curl} \mathbf{u}_{H}\right) \cdot \boldsymbol{\gamma}_{\mathbf{t}}\left(\mathbf{v}_{H}\right) d \tau \\
& +\sum_{T \in \mathcal{T}_{H}(\Omega)} \int_{\partial T \cap \Omega} \bar{\alpha}^{-1} \alpha_{T} \alpha_{T^{\prime}} h_{F}^{-1} \boldsymbol{\gamma}_{\mathbf{t}}\left(\mathbf{u}_{H}\right) \cdot \boldsymbol{\gamma}_{\mathbf{t}}\left(\mathbf{v}_{H}\right) d \tau \\
& -\sum_{T \in \mathcal{T}_{H}(\Omega)} \int_{\partial T \cap \Omega} \bar{\alpha}^{-1} \alpha_{T} \boldsymbol{\gamma}_{\mathbf{t}}\left(\mathbf{u}_{H}^{+}\right) \cdot \boldsymbol{\pi}_{\mathbf{t}}\left(\mu^{-1} \operatorname{curl} \mathbf{v}_{H}\right) d \tau \\
& +\sum_{T \in \mathcal{T}_{H}(\Omega)} \int_{\partial T \cap \Gamma} \alpha_{T}^{-1} h_{F} \boldsymbol{\pi}_{\mathbf{t}}\left(\mu^{-1} \mathbf{c u r l} \mathbf{u}_{H}\right) \cdot \boldsymbol{\pi}_{\mathbf{t}}\left(\mu^{-1} \operatorname{curl} \mathbf{v}_{H}\right) d \tau, \\
& \tilde{b}_{H}\left(\tilde{\boldsymbol{\lambda}}_{H}, \mathbf{v}_{H}\right):=-\sum_{F \in \mathcal{F}_{H}(\Omega)} \int_{F} \tilde{\boldsymbol{\lambda}}_{H} \cdot\left[\boldsymbol{\pi}_{\mathbf{t}}\left(\mu^{-1} \operatorname{curl} \mathbf{v}_{H}\right)\right] d \tau, \\
& \tilde{d}_{H}\left(\tilde{\boldsymbol{\lambda}}_{H}, \boldsymbol{\mu}_{H}\right):=\sum_{F \in \mathcal{F}_{H}(\Omega)} \int_{F} \bar{\alpha} h_{F}^{-1} \tilde{\boldsymbol{\lambda}}_{H} \cdot \boldsymbol{\mu}_{H} d \tau .
\end{aligned}
$$

The variational system (3.24) represents a symmetric saddle point problem which can be solved as in the standard mortar approach. Denoting by $\tilde{\mathbf{A}}_{H}, \tilde{\mathbf{B}}_{H}, \tilde{\mathbf{D}}_{H}$ the matrices and by $\mathbf{b}_{H}$ the vector associated with the bilinear forms and the right-hand side in the first equation of (3.24), the algebraic form of the saddle point problem is

$$
\left(\begin{array}{cc}
\tilde{\mathbf{A}}_{H} & \tilde{\mathbf{B}}_{H} \\
\tilde{\mathbf{B}}_{H}^{T} & -\tilde{\mathbf{D}}_{H}
\end{array}\right)\left(\begin{array}{c}
\mathbf{u}_{H} \\
\tilde{\boldsymbol{\lambda}}_{H}
\end{array}\right)=\left(\begin{array}{c}
\mathbf{b}_{H} \\
\mathbf{0}
\end{array}\right)
$$

Static condensation of $\mathbf{u}_{H}$ results in the equivalent Schur complement system

$$
\left(\tilde{\mathbf{D}}_{H}+\tilde{\mathbf{B}}_{H}^{T} \tilde{\mathbf{A}}_{H}^{-1} \tilde{\mathbf{B}}_{H}\right) \tilde{\boldsymbol{\lambda}}_{H}=\tilde{\mathbf{B}}_{H}^{T} \tilde{\mathbf{A}}_{H}^{-1} \mathbf{b}_{H}
$$

4. A posteriori error analysis. The residual a posteriori error estimator for the symmetric IPDG-H method (3.15a)-(3.15c) is given by

$$
\begin{aligned}
\eta:= & \left(\sum_{T \in \mathcal{T}_{H}(\Omega)}\left(\eta_{T, 1}^{2}+\eta_{T, 2}^{2}+\eta_{T, 3}^{2}\right)+\sum_{F \in \mathcal{F}_{H}(\Omega)}\left(\eta_{F, 1}^{2}+\eta_{F, 2}^{2}\right)\right. \\
& \left.+\sum_{F \in \mathcal{F}_{H}\left(\Gamma_{N}\right)}\left(\eta_{F, 3}^{2}+\eta_{F, 4}^{2}\right)\right)^{1 / 2} .
\end{aligned}
$$

They consist of the element residuals

$$
\begin{aligned}
& \eta_{T, 1}:=\left\|\mu \mathbf{p}_{H}-\operatorname{curl} \mathbf{u}_{H}\right\|_{0, T} \quad \text { for all } T \in \mathcal{T}_{H}(\Omega), \\
& \eta_{T, 2}:=h_{T}\left\|\mathbf{f}-\mathbf{c u r l} \mathbf{p}_{H}-\sigma \mathbf{u}_{H}\right\|_{0, T} \quad \text { for all } T \in \mathcal{T}_{H}(\Omega), \\
& \eta_{T, 3}:=h_{T}\left\|\nabla \cdot\left(\mathbf{f}-\sigma \mathbf{u}_{H}\right)\right\|_{0, T} \quad \text { for all } T \in \mathcal{T}_{H}(\Omega),
\end{aligned}
$$


and the face residuals

$$
\begin{aligned}
\eta_{F, 1} & :=h_{F}^{1 / 2}\left\|\left[\boldsymbol{\pi}_{\mathbf{t}}\left(\mathbf{p}_{H}\right)\right]\right\|_{0, F} \quad \text { for all } F \in \mathcal{F}_{H}(\Omega), \\
\eta_{F, 2} & :=h_{F}^{1 / 2}\left\|\mathbf{n}_{F} \cdot\left[\mathbf{f}-\sigma \mathbf{u}_{H}\right]\right\|_{0, F} \quad \text { for all } F \in \mathcal{F}_{H}(\Omega), \\
\eta_{F, 3} & :=h_{F}^{1 / 2}\left\|\mathbf{g}_{2}-\boldsymbol{\pi}_{\mathbf{t}}\left(\mathbf{p}_{H}\right)\right\|_{0, F} \quad \text { for all } F \in \mathcal{F}_{H}\left(\Gamma_{N}\right), \\
\eta_{F, 4} & :=h_{F}^{1 / 2}\left\|\mathbf{n}_{F} \cdot\left(\mathbf{f}-\sigma \mathbf{u}_{H}\right)\right\|_{0, F} \quad \text { for all } F \in \mathcal{F}_{H}\left(\Gamma_{N}\right) .
\end{aligned}
$$

The nonconformity of the symmetric IPDG-H method results in some consistency error

$$
\xi:=\min _{\tilde{\mathbf{v}}_{H} \in \mathbf{V}}\left(\sum_{T \in \mathcal{T}_{H}(\Omega)}\left(\left\|\mathbf{u}_{H}-\tilde{\mathbf{v}}_{H}\right\|_{0, T}^{2}+\left\|\operatorname{curl}\left(\mathbf{u}_{H}-\tilde{\mathbf{v}}_{H}\right)\right\|_{0, T}^{2}\right)\right)^{1 / 2}
$$

with the unique minimizer $\tilde{\mathbf{u}}_{H} \in \mathbf{V}$ of $(4.4)$ and $\xi^{2}=\left\|\mathbf{u}_{H}-\tilde{\mathbf{u}}_{H}\right\|_{0, \Omega}^{2}+\| \operatorname{curl}\left(\mathbf{u}_{H}-\right.$ $\left.\tilde{\mathbf{u}}_{H}\right) \|_{0, \Omega}^{2}$.

Theorem 4.1. Let $(\mathbf{p}, \mathbf{u}) \in \mathbf{Q} \times \mathbf{V}$ and $\left(\mathbf{p}_{H}, \mathbf{u}_{H}, \boldsymbol{\lambda}_{H}\right) \in \mathbf{Q}_{H} \times \mathbf{V}_{H} \times \mathbf{M}_{H}$ be the solutions of (3.5) and (3.15a)-(3.15c), let $\eta$ and $\xi$ be the residual error estimator and the consistency error of (4.1) and (4.4). Then,

$$
\left\|(\mathbf{u}, \mathbf{p})-\left(\mathbf{u}_{H}, \mathbf{p}_{H}\right)\right\|:=\left(\left\|\mathbf{p}-\mathbf{p}_{H}\right\|_{\mathbf{Q}}^{2}+\left\|\mathbf{u}-\mathbf{u}_{H}\right\|_{c u r l, H, \Omega}^{2}\right)^{1 / 2} \lesssim \eta+\xi
$$

We will provide the proof of Theorem 4.1 by a series of lemmas. We assume $\left(\tilde{\mathbf{p}}_{H}, \tilde{\mathbf{u}}_{H}\right) \in \mathbf{Q} \times \mathbf{V}$ to be some approximation of the solution $(\mathbf{p}, \mathbf{u}) \in \mathbf{Q} \times \mathbf{V}$ of the mixed problem (3.5) obtained by means of the solution $\left(\mathbf{p}_{H}, \mathbf{u}_{H}, \boldsymbol{\lambda}_{H}\right)$ of the symmetric IPDG-H method (3.15a)-(3.15c). It is an immediate consequence of Theorem 3.1 that the error $\left(\mathbf{p}-\tilde{\mathbf{p}}_{H}, \mathbf{u}-\tilde{\mathbf{u}}_{H}\right)$ satisfies

$$
\left\|\left(\mathbf{p}-\tilde{\mathbf{p}}_{H}, \mathbf{u}-\tilde{\mathbf{u}}_{H}\right)\right\|_{\mathbf{Q} \times \mathbf{V}} \lesssim\left\|\operatorname{Res}_{1}\right\|_{\mathbf{Q}^{*}}+\left\|\operatorname{Res}_{2}\right\|_{\mathbf{V}_{0}^{*}}
$$

with residuals $\operatorname{Res}_{1} \in \mathbf{Q}^{*}$ and $\operatorname{Res}_{2} \in \mathbf{V}_{0}^{*}$,

$$
\begin{array}{ll}
\operatorname{Res}_{1}(\mathbf{q}):=\ell^{(1)}(\mathbf{q})-a\left(\tilde{\mathbf{p}}_{H}, \mathbf{q}\right)+b\left(\tilde{\mathbf{u}}_{H}, \mathbf{q}\right) & \text { for } \mathbf{q} \in \mathbf{Q}, \\
\operatorname{Res}_{2}(\mathbf{v}):=\ell^{(2)}(\mathbf{v})-b\left(\mathbf{v}, \tilde{\mathbf{p}}_{H}\right)-c\left(\tilde{\mathbf{u}}_{H}, \mathbf{v}\right) & \text { for } \mathbf{v} \in \mathbf{V}_{0} .
\end{array}
$$

LEMMA 4.1. Let $\left(\mathbf{p}_{H}, \mathbf{u}_{H}, \boldsymbol{\lambda}_{H}\right) \in \mathbf{Q}_{H} \times \mathbf{V}_{H} \times \mathbf{M}_{H}$ be the solution of (3.15a)(3.15c) with the numerical flux $\hat{\mathbf{p}}_{H}$ from (3.18). The choice of $\tilde{\mathbf{p}}_{H}=\mathbf{p}_{H}$ and of $\tilde{\mathbf{u}}_{H} \in \mathbf{V}$ as the unique minimizer of (4.4) imply

$$
\left\|R e s_{1}\right\|_{Q^{*}} \lesssim\left(\sum_{T \in \mathcal{T}_{H}(\Omega)} \eta_{T, 1}^{2}\right)^{1 / 2}+\xi
$$

Proof. With the $\mathbf{L}^{2}$-projection $\mathbf{q}_{H}=\mathbf{P}_{\mathbf{Q}_{H}} \mathbf{q}$ of $\mathbf{q} \in \mathbf{Q}$ onto $\mathbf{Q}_{H}$, we have $\left\|\mathbf{q}_{H}\right\|_{0, \Omega} \leq\|\mathbf{q}\|_{0, \Omega}$ and

$$
\operatorname{Res}_{1}(\mathbf{q})=\operatorname{Res}_{1}\left(\mathbf{q}-\mathbf{P}_{\mathbf{Q}_{H}} q\right)+\operatorname{Res}_{1}\left(\mathbf{P}_{\mathbf{Q}_{H}} q\right) .
$$


In view of $(4.7 \mathrm{a})$ and $(3.3 \mathrm{a}),(3.3 \mathrm{~b}),(3.3 \mathrm{~d})$, it follows that

$$
\begin{aligned}
\operatorname{Res}_{1}\left(\mathbf{q}-\mathbf{P}_{\mathbf{Q}_{H}} \mathbf{q}\right)= & \sum_{T \in \mathcal{T}_{H}(\Omega)} \int_{T}\left(\operatorname{curl} \mathbf{u}_{H}-\mu \mathbf{p}_{H}\right) \cdot\left(\mathbf{q}-\mathbf{P}_{\mathbf{Q}_{H}} \mathbf{q}\right) d x \\
& +\sum_{T \in \mathcal{T}_{H}(\Omega)} \int_{T} \operatorname{curl}\left(\tilde{\mathbf{u}}_{H}-\mathbf{u}_{H}\right) \cdot\left(\mathbf{q}-\mathbf{P}_{\mathbf{Q}_{H}} \mathbf{q}\right) d x
\end{aligned}
$$

Straightforward estimation and $\left\|\mathbf{q}-\mathbf{P}_{\mathbf{Q}_{H}} \mathbf{q}\right\|_{0, \Omega} \leq\|\mathbf{q}\|_{\mathbf{Q}}$ yield

$$
\begin{aligned}
& \left|\operatorname{Res}_{1}\left(\mathbf{q}-\mathbf{P}_{\mathbf{Q}_{H}} \mathbf{q}\right)\right| \leq\left(\left(\sum_{T \in \mathcal{T}_{H}(\Omega)}\left\|\mathbf{c u r l} \mathbf{u}_{H}-\mu \mathbf{p}_{H}\right\|_{0, T}^{2}\right)^{1 / 2}\right. \\
& \left.+\left(\sum_{T \in \mathcal{T}_{H}(\Omega)}\left\|\operatorname{curl}\left(\tilde{\mathbf{u}}_{H}-\mathbf{u}_{H}\right)\right\|_{0, T}^{2}\right)^{1 / 2}\right)\left(\sum_{T \in \mathcal{T}_{H}(\Omega)}\left\|\mathbf{q}-\mathbf{P}_{\mathbf{Q}} \mathbf{q}\right\|_{0, T}^{2}\right)^{1 / 2} \\
& \leq\left(\left(\sum_{T \in \mathcal{T}_{H}(\Omega)} \eta_{T, 1}^{2}\right)^{1 / 2}+\xi\right)\|\mathbf{q}\|_{Q} .
\end{aligned}
$$

Similar arguments for the last term in (4.9) and $\left\|\mathbf{P}_{\mathbf{Q}_{H}} \mathbf{q}\right\|_{0, \Omega} \leq\|\mathbf{q}\|_{0, \Omega}$ reveal

$$
\begin{aligned}
\left|\operatorname{Res}_{1}\left(\mathbf{P}_{\mathbf{Q}_{H}} \mathbf{q}\right)\right| \leq & \left(\sum_{T \in \mathcal{T}_{H}(\Omega)}\left\|\operatorname{curl} \mathbf{u}_{H}-\mu \mathbf{p}_{H}\right\|_{0, T}^{2}\right)^{1 / 2}\left\|\mathbf{P}_{\mathbf{Q}_{H}} q\right\|_{0, \Omega} \\
& +\left(\sum_{T \in \mathcal{T}_{H}(\Omega)}\left\|\operatorname{curl}\left(\hat{\mathbf{u}}_{H}-\mathbf{u}_{H}\right)\right\|_{0, T}^{2}\right)^{1 / 2}\left\|\mathbf{P}_{\mathbf{Q}_{H}} q\right\|_{0, \Omega} \\
& \leq\left(\left(\sum_{T \in \mathcal{T}_{H}(\Omega)} \eta_{T, 1}^{2}\right)^{1 / 2}+\xi\right)\|\mathbf{q}\|_{Q} .
\end{aligned}
$$

The combination of (4.10) and (4.11) concludes the proof. $\square$

LEMma 4.2. For $\tilde{\mathbf{p}}_{H}=\mathbf{p}_{H}$ and some approximation $\tilde{\mathbf{u}}_{H} \in \mathbf{V}_{H}$ let the residual Res $_{2}$ of $(4.7 \mathrm{~b})$ satisfy

$$
\mathbf{N d}_{0 ; \Gamma_{D}}^{\mathbf{1}}\left(\Omega ; \mathcal{T}_{H}(\Omega)\right) \subset \operatorname{Ker} \operatorname{Res}_{2}
$$

Then, it holds

$$
\begin{gathered}
\left\|R e s_{2}\right\|_{V_{0}^{*}} \lesssim\left(\sum_{T \in \mathcal{T}_{H}(\Omega)}\left(\eta_{T, 2}^{2}+\eta_{T, 3}^{2}\right)+\sum_{F \in \mathcal{F}_{H}(\Omega)}\left(\eta_{F, 1}^{2}+\eta_{F, 2}^{2}\right)\right. \\
\left.+\sum_{F \in \mathcal{F}_{H}\left(\Gamma_{N}\right)}\left(\eta_{F, 3}^{2}+\eta_{F, 4}^{2}\right)\right)^{1 / 2}+\xi .
\end{gathered}
$$

Proof. Given any $\mathbf{v} \in \mathbf{V}$, Theorem 1 in [48] shows that there exist $\mathbf{v}_{H} \in$ $\mathbf{N d}_{0 ; \Gamma_{D}}^{\mathbf{1}}\left(\Omega ; \mathcal{T}_{H}(\Omega)\right), \varphi \in H_{0, \Gamma_{D}}^{1}(\Omega)$, and $\mathbf{z} \in\left(H_{0, \Gamma_{D}}^{1}(\Omega)\right)^{3}$ such that

$$
\mathbf{v}-\mathbf{v}_{H}=\nabla \varphi+\mathbf{z}
$$


and with appropriate patches $\omega_{T}$ and $\omega_{F}$

$$
\begin{aligned}
\|\varphi\|_{0, T} & \lesssim h_{T}\|\mathbf{v}\|_{c u r l ; \omega_{T}} \quad \text { for } T \in \mathcal{T}_{H}(\Omega), \\
\|\nabla \varphi\|_{0, T} & \lesssim\|\mathbf{v}\|_{c u r l ; \omega_{T}} \quad \text { for } T \in \mathcal{T}_{H}(\Omega), \\
h_{F}^{-1 / 2}\|\varphi\|_{0, F} & \lesssim\|\mathbf{v}\|_{c u r l ; \omega_{F}} \quad \text { for } F \in \mathcal{F}_{H}\left(\Omega \cup \Gamma_{N}\right), \\
\|\mathbf{z}\|_{0, T} & \lesssim h_{T}\|\mathbf{v}\|_{c u r l ; \Omega} \quad \text { for } T \in \mathcal{T}_{H}(\Omega), \\
h_{F}^{-1 / 2}\left\|\gamma_{\mathbf{t}}(\mathbf{z})\right\|_{0, F} & \lesssim\|\mathbf{v}\|_{0, \omega_{F}} \quad \text { for } F \in \mathcal{F}_{H}\left(\Omega \cup \Gamma_{N}\right) .
\end{aligned}
$$

It is a consequence of (4.12) and (4.14) that

$$
\operatorname{Res}_{\mathbf{2}}(\mathbf{v})=\operatorname{Res}_{\mathbf{2}}\left(\mathbf{v}-\mathbf{v}_{h}\right)=\operatorname{Res}_{\mathbf{2}}(\nabla \varphi)+\operatorname{Res}_{\mathbf{2}}(\mathbf{z})
$$

The first term on the right-hand side in (4.16) reads

$$
\begin{gathered}
\operatorname{Res}_{2}(\nabla \varphi)=\sum_{T \in \mathcal{T}_{H}(\Omega)} \int_{T} \mathbf{f} \cdot \nabla \varphi d x+\sum_{F \in \mathcal{F}_{H}\left(\Gamma_{N}\right)} \int_{F} \mathbf{g}_{2} \cdot \gamma_{\mathbf{t}}(\nabla \varphi) d \tau \\
-\sum_{T \in \mathcal{T}_{H}(\Omega)} \int_{T} \sigma \mathbf{u}_{H} \cdot \nabla \varphi d x-\sum_{T \in \mathcal{T}_{H}(\Omega)} \int_{T} \sigma\left(\tilde{\mathbf{u}}_{H}-\mathbf{u}_{H}\right) \cdot \nabla \varphi d x .
\end{gathered}
$$

An application of Green's formula gives

$$
\begin{aligned}
& \sum_{T \in \mathcal{T}_{H}(\Omega)} \int_{T}\left(\mathbf{f}-\sigma \mathbf{u}_{H}\right) \cdot \nabla \varphi d x=-\sum_{T \in \mathcal{T}_{H}(\Omega)} \int_{T} \nabla \cdot\left(\mathbf{f}-\sigma \mathbf{u}_{H}\right) \varphi d x \\
& +\sum_{F \in \mathcal{F}_{H}(\Omega)} \int_{F} \mathbf{n}_{F} \cdot\left[\mathbf{f}-\sigma \mathbf{u}_{H}\right] \varphi d \tau+\sum_{F \in \mathcal{F}_{H}\left(\Gamma_{N}\right)} \int_{F} \mathbf{n}_{F} \cdot\left(\mathbf{f}-\sigma \mathbf{u}_{H}\right) \varphi d \tau .
\end{aligned}
$$

Since $\left.\gamma_{\mathbf{t}}(\nabla \varphi)\right|_{F}=\operatorname{curl}_{F} \varphi$ on $F \in \mathcal{F}_{H}\left(\Gamma_{N}\right)$, a further application of Stokes' formula yields

$$
\begin{aligned}
& \sum_{F \in \mathcal{F}_{H}\left(\Gamma_{N}\right)} \int_{F} \mathbf{g}_{2} \cdot \gamma_{\mathbf{t}}(\nabla \varphi) d \tau=\sum_{F \in \mathcal{F}_{H}\left(\Gamma_{N}\right)} \int_{F} \mathbf{g}_{2} \cdot \operatorname{curl}_{F} \varphi d \tau \\
= & \sum_{F \in \mathcal{F}_{H}\left(\Gamma_{N}\right)} \int_{F} \operatorname{curl}_{F} \mathbf{g}_{2} \varphi d \tau-\sum_{E \in \mathcal{E}_{H}\left(\Gamma_{N}\right)} \int_{E}\left(\mathbf{t}_{E} \cdot\left\{\mathbf{g}_{2}\right\}[\varphi]+\mathbf{t}_{E} \cdot\left[\mathbf{g}_{2}\right]\{\varphi\}\right) d s \\
- & \sum_{E \in \mathcal{E}_{H}\left(\partial \Gamma_{N}\right)} \int_{E} \mathbf{t}_{E} \cdot \mathbf{g}_{2} \varphi d s .
\end{aligned}
$$

Since $\mathbf{g}_{2} \in \mathbf{H}\left(\operatorname{curl}_{\Gamma_{N}}^{0} ; \Gamma_{N}\right)$, we have $\operatorname{curl}_{F} \mathbf{g}_{2}=0, F \in \mathcal{F}_{H}\left(\Gamma_{N}\right)$. Since $\varphi \in H_{0, \Gamma_{D}}^{1}(\Omega)$, we have $\varphi=0$ on $E \in \mathcal{E}_{H}\left(\overline{\Gamma_{N}}\right)$. Consequently, (4.19) yields

$$
\sum_{F \in \mathcal{F}_{H}\left(\Gamma_{N}\right)} \int_{F} \mathbf{g}_{2} \cdot \gamma_{\mathbf{t}}(\nabla \varphi) d \tau=0
$$


With (4.18) and (4.20), (4.17) leads to

$$
\begin{aligned}
& \left|\operatorname{Res}_{2}(\nabla \varphi)\right| \lesssim\left(\sum_{T \in \mathcal{T}_{H}(\Omega)} h_{T}^{2} \| \nabla \cdot\left(\mathbf{f}-\sigma \mathbf{u}_{H} \|_{0, T}^{2}\right)^{1 / 2}\left(\sum_{T \in \mathcal{T}_{H}(\Omega)} h_{T}^{-2}\|\varphi\|_{0, T}^{2}\right)^{1 / 2}\right. \\
& +\left(\sum_{F \in \mathcal{F}_{H}(\Omega)} h_{F}\left\|\mathbf{n}_{F} \cdot\left[\mathbf{f}-\sigma \mathbf{u}_{H}\right]\right\|_{0, F}^{2}\right)^{1 / 2}\left(\sum_{F \in \mathcal{F}_{H}(\Omega)} h_{F}^{-1}\|\varphi\|_{0, F}^{2}\right)^{1 / 2} \\
& +\left(\sum_{F \in \mathcal{F}_{H}\left(\Gamma_{N}\right)} h_{F}\left\|\mathbf{n}_{F} \cdot\left(\mathbf{f}-\sigma \mathbf{u}_{H}\right)\right\|_{0, F}^{2}\right)^{1 / 2}\left(\sum_{F \in \mathcal{F}_{H}\left(\Gamma_{N}\right)} h_{F}^{-1}\|\varphi\|_{0, F}^{2}\right)^{1 / 2} \\
& +\left(\sum_{T \in \mathcal{T}_{H}(\Omega)}\left\|\tilde{\mathbf{u}}_{H}-\mathbf{u}_{H}\right\|_{0, T}^{2}\right)^{1 / 2}\left(\sum_{T \in \mathcal{T}_{H}(\Omega)}\|\nabla \varphi\|_{0, T}^{2}\right)^{1 / 2} .
\end{aligned}
$$

This and (4.15a)-(4.15c) imply

$$
\begin{aligned}
\left|\operatorname{Res}_{2}(\nabla \varphi)\right| \lesssim( & \left(\sum_{T \in \mathcal{T}_{H}(\Omega)} \eta_{T, 3}^{2}\right)^{1 / 2}+\left(\sum_{F \in \mathcal{F}_{H}(\Omega)} \eta_{F, 2}^{2}\right)^{1 / 2} \\
& \left.+\left(\sum_{F \in \mathcal{F}_{H}\left(\Gamma_{N}\right)} \eta_{F, 4}^{2}\right)^{1 / 2}+\xi\right)\|\mathbf{v}\|_{\text {curl } ; \Omega} .
\end{aligned}
$$

On the other hand, the second term on the right-hand side of (4.16) reads

$$
\begin{aligned}
\operatorname{Res}_{2}(\mathbf{z})= & \sum_{T \in \mathcal{T}_{H}(\Omega)} \int_{T} \mathbf{f} \cdot \mathbf{z} d x+\sum_{F \in \mathcal{F}_{H}\left(\Gamma_{N}\right)} \int_{F} \mathbf{g}_{2} \cdot \gamma_{\mathbf{t}}(\mathbf{z}) d \tau \\
& -\sum_{T \in \mathcal{T}_{H}(\Omega)} \int_{T} \mathbf{p}_{H} \cdot \mathbf{c u r l} \mathbf{z} d x-\sum_{T \in \mathcal{T}_{H}(\Omega)} \int_{T} \sigma \mathbf{u}_{H} \cdot \mathbf{z} d x \\
& -\sum_{T \in \mathcal{T}_{H}(\Omega)} \int_{T} \sigma\left(\tilde{\mathbf{u}}_{H}-\mathbf{u}_{H}\right) \cdot \mathbf{z} d x .
\end{aligned}
$$

Since $\left[\gamma_{\mathbf{t}}(\mathbf{z})\right]=0$ on $F \in \mathcal{F}_{H}(\Omega)$, an application of Stokes' theorem gives

$$
\begin{aligned}
& \sum_{T \in \mathcal{T}_{H}(\Omega)} \int_{T} \mathbf{p}_{H} \cdot \operatorname{curl} \mathbf{z} d x=\sum_{T \in \mathcal{T}_{H}(\Omega)} \int_{T} \operatorname{curl} \mathbf{p}_{H} \cdot \mathbf{z} d x \\
& +\sum_{F \in \mathcal{F}_{H}(\Omega)} \int_{F}\left[\boldsymbol{\pi}_{\mathbf{t}}\left(\mathbf{p}_{H}\right)\right] \cdot \gamma_{\mathbf{t}}(\mathbf{z}) d \tau+\sum_{F \in \mathcal{F}_{H}\left(\Gamma_{N}\right)} \int_{F} \boldsymbol{\pi}_{\mathbf{t}}\left(\mathbf{p}_{H}\right) \cdot \gamma_{\mathbf{t}}(\mathbf{z}) d \tau .
\end{aligned}
$$

This and (4.22) lead to

$$
\begin{aligned}
& \operatorname{Res}_{2}(\mathbf{z})=\sum_{T \in \mathcal{T}_{H}(\Omega)} \int_{T}\left(\mathbf{f}-\mathbf{c u r l} \mathbf{p}_{H}-\sigma \mathbf{u}_{H}\right) \cdot \mathbf{z} d x \\
& -\sum_{F \in \mathcal{F}_{H}(\Omega)} \int_{F}\left[\boldsymbol{\pi}_{\mathbf{t}}\left(\mathbf{p}_{H}\right)\right] \cdot \boldsymbol{\gamma}_{\mathbf{t}}(\mathbf{z}) d \tau+\sum_{F \in \mathcal{F}_{H}\left(\Gamma_{N}\right)} \int_{F}\left(\mathbf{g}_{2}-\boldsymbol{\pi}_{\mathbf{t}}\left(\mathbf{p}_{H}\right)\right) \cdot \boldsymbol{\gamma}_{\mathbf{t}}(\mathbf{z}) d \tau \\
& -\sum_{T \in \mathcal{T}_{H}(\Omega)} \int_{T} \sigma\left(\tilde{\mathbf{u}}_{H}-\mathbf{u}_{H}\right) \cdot \mathbf{z} d x
\end{aligned}
$$


Hence, $\operatorname{Res}_{2}(\mathbf{z})$ is bounded from above by

$$
\begin{aligned}
& \left|\operatorname{Res}_{2}(\mathbf{z})\right| \lesssim \\
& \lesssim\left(\sum_{T \in \mathcal{T}_{H}(\Omega)} h_{T}^{2}\left\|\mathbf{f}-\mathbf{c u r l} \mathbf{p}_{H}-\sigma \mathbf{u}_{H}\right\|_{0, T}^{2}\right)^{1 / 2}\left(\sum_{T \in \mathcal{T}_{H}(\Omega)} h_{T}^{-2}\|\mathbf{z}\|_{0, T}^{2}\right)^{1 / 2} \\
& +\left(\sum_{F \in \mathcal{F}_{H}(\Omega)} h_{F}\left\|\left[\boldsymbol{\pi}_{\mathbf{t}}\left(\mathbf{p}_{H}\right)\right]\right\|_{0, F}^{2}\right)^{1 / 2}\left(\sum_{F \in \mathcal{F}_{H}(\Omega)} h_{F}^{-1}\left\|\boldsymbol{\gamma}_{\mathbf{t}}(\mathbf{z})\right\|_{0, F}^{2}\right)^{1 / 2} \\
& +\left(\sum_{F \in \mathcal{F}_{H}\left(\Gamma_{N}\right)} h_{F}\left\|\mathbf{g}_{2}-\boldsymbol{\pi}_{\mathbf{t}}\left(\mathbf{p}_{H}\right)\right\|_{0, F}^{2}\right)^{1 / 2}\left(\sum_{F \in \mathcal{F}_{H}\left(\Gamma_{N}\right)} h_{F}\left\|\boldsymbol{\gamma}_{\mathbf{t}}(\mathbf{z})\right\|_{0, F}^{2}\right)^{1 / 2} \\
& +\left(\sum_{T \in \mathcal{T}_{H}(\Omega)} h_{T}^{2}\left\|\tilde{\mathbf{u}}_{H}-\mathbf{u}_{H}\right\|_{0, T}^{2}\right)^{1 / 2}\left(\sum_{T \in \mathcal{T}_{H}(\Omega)} h_{T}^{-2}\|\mathbf{z}\|_{0, T}^{2}\right)^{1 / 2} .
\end{aligned}
$$

This and $(4.15 \mathrm{~d}),(4.15 \mathrm{e})$ result in

$$
\begin{aligned}
\left|\operatorname{Res}_{2}(\mathbf{z})\right| \lesssim & \left(\left(\sum_{T \in \mathcal{T}_{H}(\Omega)} \eta_{T, 2}^{2}\right)^{1 / 2}+\left(\sum_{F \in \mathcal{F}_{H}(\Omega)} \eta_{F, 1}^{2}\right)^{1 / 2}\right. \\
& \left.+\left(\sum_{F \in \mathcal{F}_{H}\left(\Gamma_{N}\right)} \eta_{F, 3}^{2}\right)^{1 / 2}+\xi\right)\|\mathbf{v}\|_{\text {curl } ; \Omega} .
\end{aligned}
$$

The combination of (4.21) and (4.23) plus (4.16) concludes the proof.

LEMMA 4.3. For $\mathbf{v}_{H} \in \mathbf{N d}_{0, \Gamma_{D}}^{\mathbf{p}}\left(\Omega ; \mathcal{T}_{H}(\Omega)\right)$ it holds

$$
\operatorname{Res}_{2}\left(\mathbf{v}_{H}\right)=c_{H}\left(\mathbf{u}_{H}-\tilde{\mathbf{u}}_{H}, \mathbf{v}_{H}\right) \text {. }
$$

Proof. We have

$$
\begin{aligned}
& \operatorname{Res}_{2}\left(\mathbf{v}_{H}\right)=\ell_{H}^{(2)}\left(\mathbf{v}_{H}\right)-b_{H}\left(\mathbf{v}_{H}, \mathbf{p}_{H}\right)-c_{H}\left(\tilde{\mathbf{u}}_{H}, \mathbf{v}_{H}\right) \\
& =\ell_{H}^{(2)}\left(\mathbf{v}_{H}\right)-\left(b_{H}\left(\mathbf{v}_{H}, \mathbf{p}_{H}\right)+c_{H}\left(\mathbf{u}_{H}, \mathbf{v}_{H}\right)\right)+c_{H}\left(\mathbf{u}_{H}-\tilde{\mathbf{u}}_{H}, \mathbf{v}_{H}\right) .
\end{aligned}
$$

Since $\mathbf{v}_{H} \in \mathbf{N d}_{0, \Gamma_{D}}^{\mathbf{1}}\left(\Omega ; \mathcal{T}_{H}(\Omega)\right) \subset \mathbf{V}_{H}$ is an admissible test function in (3.15b), it follows that

$$
b_{H}\left(\mathbf{v}_{H}, \mathbf{p}_{H}\right)+c_{H}\left(\mathbf{u}_{H}, \mathbf{v}_{H}\right)=\ell_{H}^{(2)}\left(\mathbf{v}_{H}\right)+d_{H}\left(\mathbf{v}_{H}, \hat{\mathbf{p}}_{H}\right) .
$$

Since (3.21a), the last term vanishes

$$
d_{H}\left(\mathbf{v}_{H}, \hat{\mathbf{p}}_{H}\right)=0 .
$$

The combination of (4.25)-(4.27) concludes the proof.

Proof of Theorem 4.1. In view of Lemma 4.2 we define

$$
\widetilde{\operatorname{Res}_{2}}(\cdot):=\operatorname{Res}_{2}(\cdot)-c_{H}\left(\mathbf{u}_{H}-\tilde{\mathbf{u}}_{H}, \cdot\right) .
$$

It follows that

$$
\left\|\operatorname{Res}_{2}\right\|_{V_{0}^{*}} \lesssim\left\|\widetilde{\operatorname{Res}_{2}}\right\|_{V_{0}^{*}}+\xi
$$


In view of (4.24), we have $\mathbf{N d}_{0, \Gamma_{D}}^{1}\left(\Omega ; \mathcal{T}_{H}(\Omega)\right) \subset$ Ker $\widetilde{\operatorname{Res}_{2}}$. Hence, Lemma 4.3 with $\operatorname{Res}_{2}$ replaced by $\widetilde{R e s} 2$ yields

$$
\begin{aligned}
\left\|\widetilde{\operatorname{Res}_{2}}\right\|_{V_{0}^{*}} \lesssim & \left(\sum_{T \in \mathcal{T}_{H}(\Omega)}\left(\eta_{T, 2}^{2}+\eta_{T, 3}^{2}\right)+\sum_{F \in \mathcal{F}_{H}(\Omega)}\left(\eta_{F, 1}^{2}+\eta_{F, 2}^{2}\right)\right. \\
& \left.+\sum_{F \in \mathcal{F}_{H}\left(\Gamma_{N}\right)}\left(\eta_{F, 3}^{2}+\eta_{F, 4}^{2}\right)\right)^{1 / 2} .
\end{aligned}
$$

As in the case of the symmetric IPDG method (cf., e.g., [20,37]), the consistency error admits the upper bound

$$
\xi \lesssim\left(\sum_{F \in \mathcal{F}_{H}(\Omega)} \eta_{F, 5}^{2}\right)^{1 / 2}, \quad \eta_{F, 5}:=h_{F}^{-1 / 2}\left\|\left[\boldsymbol{\gamma}_{\mathbf{t}}\left(\mathbf{u}_{H}\right)\right]\right\|_{0, F} .
$$

The combination of (4.8),(4.29)-(4.31) and the triangle inequality

$$
\left\|(\mathbf{u}, \mathbf{p})-\left(\mathbf{u}_{H}, \mathbf{p}_{H}\right)\right\| \leq\left\|(\mathbf{u}, \mathbf{p})-\left(\tilde{\mathbf{u}}_{H}, \mathbf{p}_{H}\right)\right\|+\left\|\mathbf{u}_{H}-\tilde{\mathbf{u}}_{H}\right\|_{c u r l, H, \Omega}
$$

conclude the proof.

\section{Numerical Results.}

5.1. The Adaptive Cycle. The adaptive IPDG-H method is realized within an adaptive cycle with the basic steps 'SOLVE', 'ESTIMATE', 'MARK', and 'REFINE'. 'SOLVE' stands for the numerical solution of the hybridized IPDG scheme with the mortar approach of section 3 implemented in the 'nudg' code from [33] for the solution of (3.24). The step 'ESTIMATE' is devoted to the computation of the element residuals $\eta_{T, i}, 1 \leq i \leq 3$, and the face residuals $\eta_{F, i}, 1 \leq i \leq 4$ (cf. (4.2a)-(4.2c) and (4.3a)-(4.3c)) as the basic constituents of the residual error estimator $\eta$ (cf. (4.1)). Moreover, the consistency error $\xi$ (cf. (4.4)) is estimated by the additional face residuals $\eta_{F, 5}$ according to (4.27). The following step 'MARK' deals with the marking of elements and faces for refinement by a bulk criterion, also known as Dörfler marking [29]. In particular, given a universal constant $0<\theta<1$, sets $\mathcal{M}_{T} \subset \mathcal{T}_{H}(\Omega) \times\{1,2,3\}$ and $\mathcal{M}_{F} \subset \mathcal{F}_{H}(\bar{\Omega}) \times\{1,2,3,4,5\}$ of almost minimal cardinality are determined such that

$$
\theta \eta^{2} \leq \sum_{(T, i) \in \mathcal{M}_{T}} \eta_{T, i}^{2}+\sum_{(F, i) \in \mathcal{M}_{F}} \eta_{F, i}^{2}
$$

The bulk criterion (5.1) is implemented by a greedy algorithm. For sufficiently small $\theta$, it is expected that the bulk criterion may yield asymptotic optimal complexity (cf., e.g., [12] in case of adaptive IPDG methods for standard second order elliptic boundary value problems). The final step 'REFINE' takes care of the practical realization of the adaptive refinement. Elements $T \in \mathcal{T}_{H}(\Omega)$ and faces $F \in \mathcal{F}_{H}(\bar{\Omega})$ such that $(T, i) \in \mathcal{M}_{T}$ for some $1 \leq i \leq 3$ and $(F, i) \in \mathcal{M}_{F}$ for some $1 \leq i \leq 5$ are refined by bisection.

5.2. Numerical Examples. For the illustration of the performance of the residual a posteriori error estimator we consider two examples of $\mathrm{H}$ (curl)-elliptic boundary value problems in $2 \mathrm{D}$ from (2.3a)-(2.3c). Both examples feature solutions in $\mathbf{H}(\mathbf{c u r l} ; \Omega)$ with components in $H^{s}(\Omega)$ for some $0<s<1$. The first one has an an 
irrotational solution on an L-shaped domain with a singularity at the reentrant corner and the second one exhibits a solenoidal solution on a circle with a cut out wedge having a singularity at the origin. For both problems, the penalty parameters in the IPDG-H method have been chosen according to $\alpha_{ \pm}:=\kappa(k+1)^{2} / 2$ with $\kappa=100$.

Example 1: We consider the L-shaped domain $\Omega:=(-1,+1)^{2} \backslash[0,+1] \times[-1,0]$ with Dirichlet boundary $\Gamma_{D}:=(0 \times(0,1) \cup(0,1) \times 0)$, Neumann boundary $\Gamma_{N}:=\Gamma \backslash \Gamma_{D}$ and data $\mu=\sigma=1$. The right-hand sides $\mathbf{f}, g_{1}, g_{2}$ in (2.3a)-(2.3c) are chosen such that

$$
\mathbf{u}=\operatorname{grad}\left(r^{2 / 3} \sin \left(\frac{2}{3} \varphi\right)\right)
$$

is the exact solution (in polar coordinates). The solution is in $\mathbf{H}(\mathbf{c u r l} ; \Omega) \cap \mathbf{H}^{2 / 3-\varepsilon}(\Omega)$ for any $\varepsilon>0$ and exhibits a singularity at the reentrant corner.

Mesh Level 0

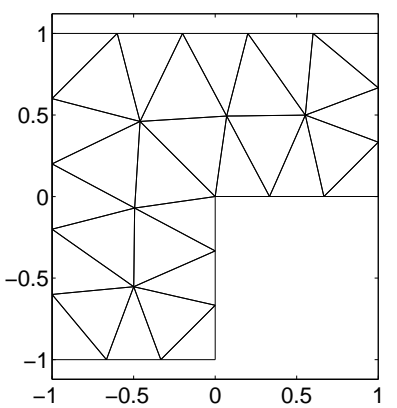

Mesh Level 8

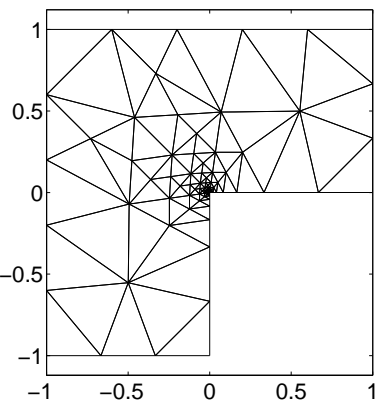

Mesh Level 18

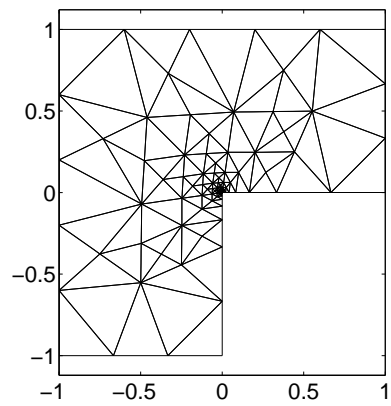

FIG. 5.1. Ex. 1: The initial mesh (left) and the meshes after 8 (middle) and 18 (right) adaptive refinement steps $(k=4$ and $\Theta=0.1)$.
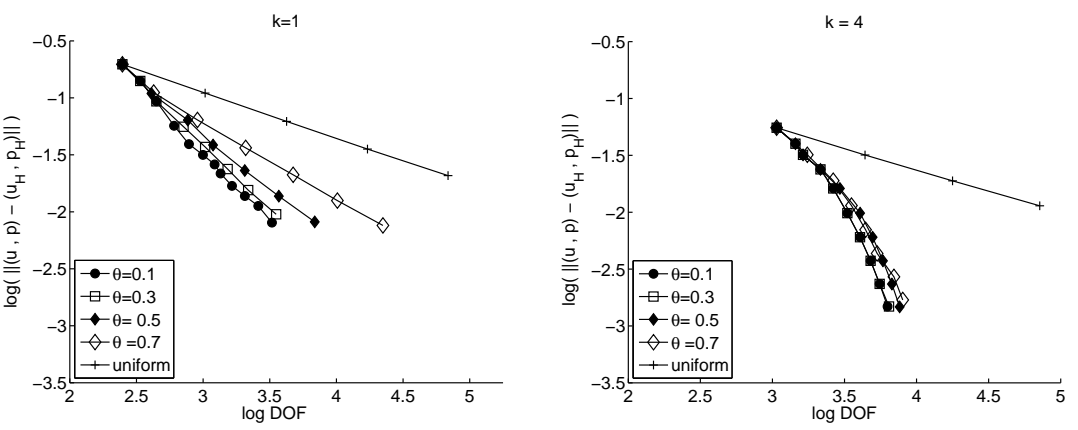

FIG. 5.2. Ex. 1: The error $\left\|(\mathbf{u}, \mathbf{p})-\left(\mathbf{u}_{H}, \mathbf{p}_{H}\right)\right\|$ versus the number of degrees of freedom on a logarithmic scale for various $\theta, k=1$ (left) and $k=4$ (right).

Figure 5.1 shows the initial mesh (left) and the meshes obtained after 8 (middle) and 18 (right) refinement steps of the adaptive algorithm in case $k=4$ and $\theta=0.1$. We observe a pronounced refinement in a vicinity of the reentrant corner. Figure 5.2 displays the global discretization error $\left\|(\mathbf{u}, \mathbf{p})-\left(\mathbf{u}_{H}, \mathbf{p}_{H}\right)\right\|(\mathrm{cf}$. (4.5)) as a function of the number of degrees of freedom (DOF) on a logarithmic scale for both uniform refinement and adaptive refinement in case $k=1$ (left) and $k=4$ (right). The 
results of the adaptive refinement are shown for various values of the constant $\theta$ in the bulk criterion (5.1). Both for $k=1$ and $k=4$ the benefits of adaptive versus uniform refinement can be clearly seen. In case $k=1$, we observe a dependence of the convergence rate on the parameter $\theta$ which is much less pronounced in case $k=4$. According to the theory for IPDG methods applied to standard second order elliptic boundary value problems (cf. [12] and the numerical results in [34]), we see that optimality is asymptotically achieved for small $\theta$.

Example 2: The domain $\Omega$ is the unit circle with a cut out wedge (see Figure 5.3). We assume $\mu=\sigma=1$ and Neumann boundary conditions on $\Gamma=\partial \Omega$. The data $\mathbf{f}$ and $g_{2}$ are chosen such that $\mathbf{u}=\operatorname{curl}\left(r^{4 / 7} \sin \left(\frac{4}{7} \varphi\right)\right)$ is the exact solution (in polar coordinates). The solution is in $\mathbf{H}(\mathbf{c u r l} ; \Omega) \cap \mathbf{H}^{4 / 7-\varepsilon}(\Omega)$ for any $\varepsilon>0$ and exhibits a singularity at the origin. We use isoparametric elements for a proper resolution of the curved part of the boundary.
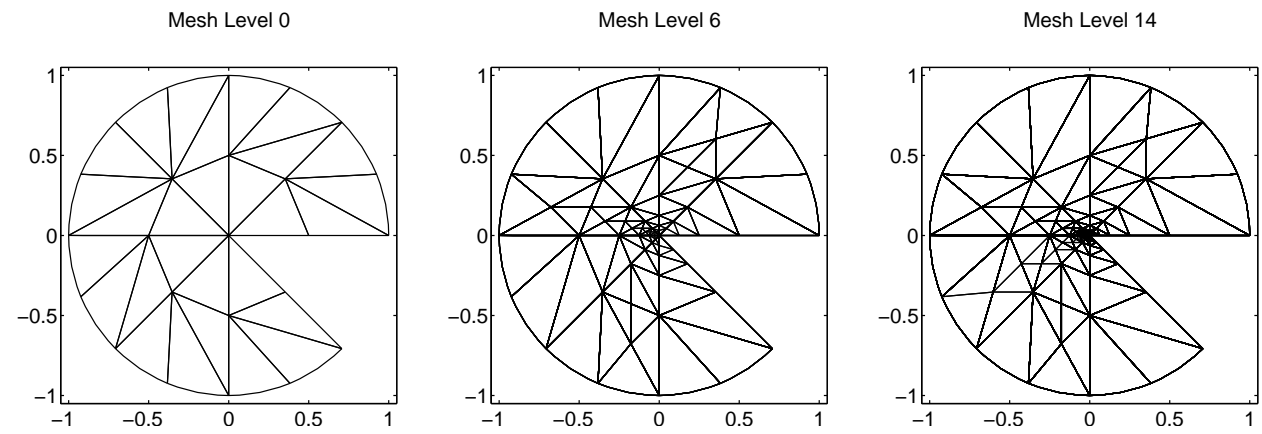

FIG. 5.3. Ex. 2: The initial mesh (left) and the meshes after 6 (middle) and 14 (right) adaptive refinement steps $(k=4$ and $\Theta=0.1)$.
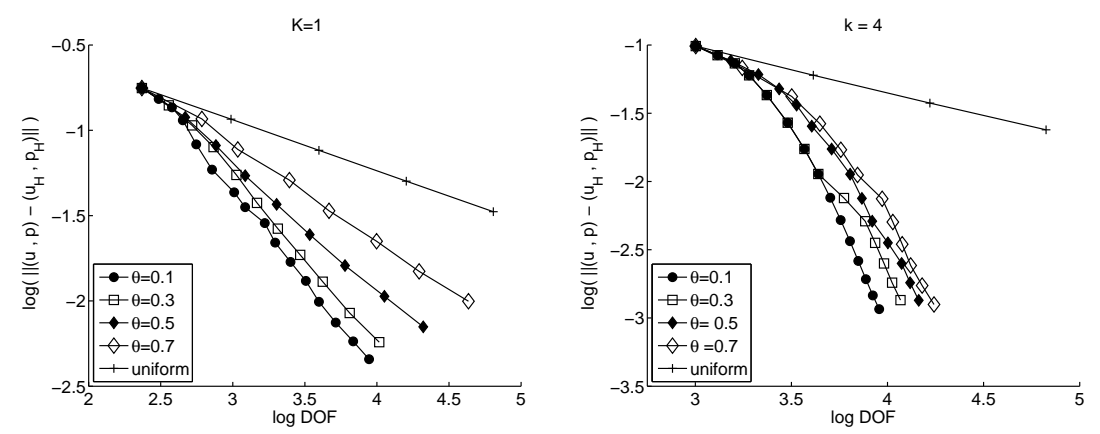

FIG. 5.4. Ex. 2: The error $\left\|(\mathbf{u}, \mathbf{p})-\left(\mathbf{u}_{H}, \mathbf{p}_{H}\right)\right\|$ versus the number of degrees of freedom on a logarithmic scale for various $\theta, k=1$ (left) and $k=4$ (right).

As for the previous example, Figures 5.3 and 5.4 display the history of the refinement process. We basically observe a similar behavior with asymptotically optimal convergence for small $\theta$. However, in the pre-asymptotic regime, the decrease of the discretization error is less pronounced. The reason is that there are two main sources for the error: the singularity at the origin and the resolution of the curved boundary. Since the error is dominated by the singularity, the greedy algorithm realizing the 
bulk criterion (5.1) picks the corresponding residuals first until those associated with the boundary resolution are taken into account.

\section{REFERENCES}

[1] M. Ainsworth, A posteriori error estimation for Discontinuous Galerkin finite element approximation. SIAM J. Numer. Anal. 45, 1777-1798, 2007.

[2] M. Ainsworth and T. Oden, A Posteriori Error Estimation in Finite Element Analysis. Wiley, Chichester, 2000.

[3] D.N. Arnold, An interior penalty finite element method with discontinuous elements. SIAM J. Numer. Anal. 19, 742-760, 1982.

[4] D.N. Arnold and F. Brezzi, Mixed and nonconforming finite element methods: implementation, postprocessing and error estimates. RAIRO Modél. Math. Anal. Numér. 19, 7-32, 1985.

[5] D.N. Arnold, F. Brezzi, B. Cockburn, and D. Marini, Unified analysis of discontinuous Galerkin methods for elliptic problems. SIAM J. Numer. Anal. 39, 1749-1779, 2002.

[6] I. Babuska and T. Strouboulis, The Finite Element Method and its Reliability. Clarendon Press, Oxford, 2001.

[7] W. Bangerth and R. Rannacher, Adaptive Finite Element Methods for Differential Equations. Lectures in Mathematics. ETH-Zürich. Birkhäuser, Basel, 2003.

[8] R. Beck, P. Deuflhard, R. Hiptmair, R.H.W. Hoppe, and B. Wohlmuth, Adaptive multilevel methods for edge element discretizations of Maxwell's equations. Surveys of Math. in Industry 8, 271-312, 1999.

[9] R. Beck, R. Hiptmair, R.H.W. Hoppe, and B. Wohlmuth, Residual based a posteriori error estimators for eddy current computation. $\mathrm{M}^{2} \mathrm{AN}$ Math. Modeling and Numer. Anal. 34, $159-182,2000$.

[10] R. Beck, R. Hiptmair, and B. Wohlmuth, Hierarchical error estimator for eddy current computation. In: Proc. 2nd European Conf. on Advanced Numer. Meth. (ENUMATH99), Jyväskylä, Finland, July 26-30, 1999 (Neittaanmäki, P. et al.; eds.), pp. 111-120, World Scientific, Singapore, 2000.

[11] R. Becker, P. Hansbo, and M.G. Larson, Energy norm a posteriori error estimation for discontinuous Galerkin methods. Comput. Methods Appl. Mech. Engrg. 192, 723-733, 2003.

[12] A. Bonito and R. Nochetto, Quasi-optimal convergence rate of an adaptive Discontinuous Galerkin method. Preprint. Department of Mathematics, University of Maryland, 2008.

[13] J.H. Bramble and J. Xu, A local post-processing technique for improving the accuracy in mixed finite element approximations. SIAM J. Numer. Anal. 26, 1267-1275, 1989.

[14] J. Brandts, Superconvergence and a posteriori error estimation for triangular mixed finite element methods. Numer. Math. 68, 311-324, 1994.

[15] F. Brezzi, J. Douglas, Jr., and L.D. Marini, Two families of mixed finite elements for second order elliptic problems. Numer. Math. 47, 217-235, 1985.

[16] A. Buffa, M. Costabel, and D. Sheen, On traces for $H(c u r l, \Omega)$ in Lipschitz domains, J. Math. Anal. Appl. 276, 845-867, 2002.

[17] C. Carstensen, A unifying theory of a posteriori finite element error control. Numer. Math. 100, 617-637, 2005.

[18] C. Carstensen, T. Gudi, and M. Jensen, A unifying theory of a posteriori error control for discontinuous Galerkin FEM. Numer. Math. 112, 363-379, 2009.

[19] C. Carstensen and R.H.W. Hoppe, Convergence analysis of an adaptive edge finite element method for the 2d eddy current equations. J. Numer. Math. 13, 19-32, 2005.

[20] C. Carstensen and R.H.W. Hoppe, Unified framework for an a posteriori error analysis of nonstandard finite element approximations of $\mathbf{H}(\mathbf{c u r l})$-elliptic problems. J. Numer. Math. 17, 27-44, 2009.

[21] C. Carstensen and J. Hu, A unifying theory of a posteriori error control for nonconforming finite element methods. Numer. Math. 107, 473-502, 2007.

[22] C. Carstensen, J. Hu, and A. Orlando, Framework for the a posteriori error analysis of nonconforming finite elements. SIAM J. Numer. Anal. 45, 68-82, 2007.

[23] P. Castillo, B. Cockburn, I. Perugia, and D. Schötzau, An a priori error estimate of the local discontinuous Galerkin method for elliptic problems. SIAM J. Numer. Anal. 38, 1676-1706, 2000 .

[24] B. Cockburn, Discontinuous Galerkin methods. Z. Angew. Math. Mech. 83, 731-754, 2003. 
[25] Cockburn, B., and Gopalakrishnan, J.; A characterization of hybridized mixed methods for second order elliptic problems. SIAM J. Numer. Anal. 42, 283-301, 2004.

[26] Cockburn, B., and Gopalakrishnan, J.; Error analysis of variable degree mixed methods for elliptic problems via hybridization. Math. Comp. 74, 1653-1677, 2005.

[27] Cockburn, B., and Gopalakrishnan, J.; New hybridization techniques. GAMM-Mitteilungen 2, 154-183 (2005).

[28] Cockburn, B., Gopalakrishnan, J., and Lazarov, R.; Unified hybridization of discontinuous Galerkin, Mixed and continuous Galerkin methods for second order elliptic problems. SIAM J. Numer. Anal. 47, 1319-1365, 2009.

[29] Dörfler, W.; A convergent adaptive algorithm for Poisson's equation. SIAM J. Numer. Anal. 33, 1106-1124, 1996.

[30] K. Eriksson, D. Estep, P. Hansbo, and C. Johnson, Computational Differential Equations. Cambridge University Press, Cambridge, 1996.

[31] B.M. Fraejis de Veubeke, Displacement and equilibrium methods in the finite element method. In: Stress Analysis (O. Zienkiewicz and G. Hollister; eds.), pp. 145-197, Wiley, New York, 1977.

[32] V. Girault and P.A. Raviart, Finite Element Approximatiuon of the Navier-Stokes Equations. Lecture Notes in Mathematics 749, Springer, Berlin-Heidelberg-New York, 1979.

[33] J.S. Hesthaven and T. Warburton, Nodal Discontinuous Galerkin Methods. Springer, BerlinHeidelberg-New York, 2008.

[34] R.H.W. Hoppe, G. Kanschat, and T. Warburton, Convergence analysis of an adaptive interior penalty discontinuous Galerkin method. SIAM J. Numer. Anal., 47, 534-550, 2009.

[35] R.H.W. Hoppe and J. Schöberl, Convergence of adaptive edge element methods for the 3D eddy currents equations. J. Comp. Math. 27, 657-676, 2009.

[36] P. Houston, I. Perugia, and D. Schötzau, Mixed discontinuous Galerkin approximation of the Maxwell operator. SIAM J. Numer. Anal. 42, 434-459, 2004.

[37] P. Houston, I. Perugia, and D. Schötzau, A posteriori error estimation for discontinuous Galerkin discretizations of $H$ (curl)-elliptic partial differential equations. IMA Journal of Numerical Analysis 27, 122-150, 2007.

[38] P. Houston, D. Schötzau, and T.P. Wihler, Energy norm a posteriori error estimation of hpadaptive discontinuous Galerkin methods for elliptic problems. Math. Models Methods Appl. Sci. 17, 33-62, 2007.

[39] G. Kanschat and R. Rannacher, Local error analysis of the interior penalty discontinuous Galerkin method for second order problems. J. Numer. Math. 10, 249-274, 2002.

[40] O. Karakashian and F. Pascal, A posteriori error estimates for a discontinuous Galerkin approximation of second-order elliptic problems. SIAM J. Numer. Anal. 41, 2374-2399, 2003.

[41] O. Karakashian and F. Pascal, Convergence of adaptive Doiscontinuous Galerkin approximations of second-order elliptic problems. SIAM J. Numer. Anal. 45, 641-665, 2007.

[42] P. Monk, Finite Element Methods for Maxwell's equations, Clarendon Press, Oxford, 2003.

[43] J.C. Nédélec, Mixed finite elements in $\mathbb{R}^{3}$, Numer. Math. 35, 315-341, 1980.

[44] P. Neittaanmäki and S. Repin, Reliable methods for mathematical modelling. Error control and a posteriori estimates. Elsevier, New York, 2004.

[45] S. Nicaise and E. Creusé, Isotropic and anisotropic a posteriori error estimation of the mixed finite element method for second order operators in divergence form. ETNA 23, 38-62, 2006.

[46] I. Perugia, D. Schötzau, and P. Monk, Stabilized interior penalty methods for the time-harmonic Maxwell equations. Comp. Meth. Appl. Mech. Engrg. 191, 4675-4697, 2002.

[47] B. Rivière and M.F. Wheeler, A posteriori error estimates and mesh adaptation strategy for discontinuous Galerkin methods applied to diffusion problems. Computers \& Mathematics with Applications 46, 141-163, 2003.

[48] J. Schöberl, A posteriori error estimates for Maxwell equations, Math. Comp. 77, 633-649, 2008.

[49] R. Verfürth, A Review of A Posteriori Estimation and Adaptive Mesh-Refinement Techniques. Wiley-Teubner, New York, Stuttgart, 1996.

[50] B. Wohlmuth and R.H.W. Hoppe, A comparison of a posteriori error estimators for mixed finite element discretizations. Math. Comp. 82, 253-279, 1999.

[51] X. Xu and R.H.W. Hoppe, On the convergence of mortar edge element methods in $\mathbf{R}^{3}$. SIAM J. Numer. Anal. 43, 1276-1294, 2005. 